\title{
ESTIMACIÓN DEL EFECTO VENTURI COMO FACTOR DESENCADENANTE DE LA PLUVIOMETRÍA EN LA SIERRA DE GRAZALEMA
}

\section{Estimation of the Venturi effect as a leading factor of pluviometry in the Grazalema Mountain}

\author{
Alfonso Jesús Naranjo-Barea ${ }^{1}$, Jesús Rodrigo-Comino ${ }^{2,3 *}$, José María Senciales-González ${ }^{1}$ \\ ${ }^{1}$ Departamento de Geografía, Universidad de Málaga. 29071, Málaga, Spain. \\ ${ }^{2}$ Instituto de Geomorfología y Suelos, Departamento de Geografía, Universidad de Málaga. 29071, Málaga, Spain. \\ ${ }^{3}$ Departamento de Geografía Física, Universidad de Trier. 54286, Trier, Germany. \\ Identificador ORCID de los autores y e-mail: \\ Alfonso Jesús Naranjo-Barea: http://orcid.org/0000-0003-3285-3457. E-mail: naranjobarea@gmail.com \\ Jesús Rodrigo-Comino: http://orcid.org/0000-0002-4823-0871. E-mail: rodrigo-comino@uma.es \\ José María Senciales-González: http://orcid.org/0000-0002-7858-1357. E-mail: senciales@uma.es \\ *Autor de contacto.
}

Recibido: 14-09-2017. Aceptado: 09-10-2017. Fecha de publicación on-line: 10/11/2017

Citation / Cómo citar este artículo: Naranjo-Barea, A. J., Rodrigo-Comino, J., Senciales-González, J. M. ${ }^{\text {a }}$ (2017). Estimación del efecto Venturi como factor desencadenante de la pluviometría en la Sierra de Grazalema. Pirineos, 172, e033. doi: http://dx.doi.org/10.3989/Pirineos.2017.172008

RESUMEN: La Sierra de Grazalema, uno de los ámbitos de la península más interesantes climatológicamente, no está lo suficientemente estudiada. Por ello, se afronta un análisis pluviométrico de detalle, con el objeto de abordar científicamente el origen de sus elevados registros pluviométricos.

Los resultados obtenidos muestran que los elevados registros pluviométricos medios anuales de Grazalema, más cuantiosos que en las estaciones situadas en la vertiente de barlovento, se deben a un efecto de depresión de sotavento (con refuerzo de efecto Venturi), producido por el Corredor del Boyar. Además, se pone de manifiesto que con la creación de nuevos puntos extrapolados georreferenciados y con valores pluviométricos, se logra obtener una cartografía pluviométrica de mayor precisión que con otros métodos, pues este mapa se ajusta mejor al relieve.

PALABRAS CLAVE: Precipitación; interpolación; cartografía pluviométrica; depresión de sotavento; Corredor del Boyar.

ABSTRACT: Climatologically, the Grazalema Mountain is one of the most interesting areas of the Iberian Peninsula. However, it has not been studied in detail. Thus, a detailed pluviometric analysis is presented by addressing the origin of its high rainfall records.

The results obtained show that the high rainfall levels on average, annually recorded in Grazalema, (more numerous than the ones recorded on the stations located at the windward slopes) are caused by a leeward depression effect (with reinforcement of Venturi effect) produced by the Corredor del Boyar. In addition, it is evidenced that with the 
creation of new georeferenced extrapolated points with rainfall values, it is possible to obtain a more accurate rainfall cartographic than with other methods, due to the fact that these maps are better suited to the relief.

KEY WORDS: Rainfall; interpolation; rainfall map; leeward depression; Corredor del Boyar.

\section{Introducción}

La Sierra de Grazalema constituye uno de los máximos pluviométricos de la península ibérica, superándose en su entorno los $2.000 \mathrm{~mm}$ de precipitación anual (Capel, 2000; Pita, 2003).

Numerosas investigaciones abordan la situación climática pasada y actual en España y Europa por sus efectos sobre la flora (Molina-Venegas et al., 2017), las actividades económicas (Draper-Munt et al., 2016; Ratcliffe et al., 2016) o el suelo (Cerdà, 1998; Imeson \& Lavee, 1998; Ruiz-Sinoga et al., 2011). Sin embargo, en concreto para la Sierra de Grazalema no existe prácticamente ningún estudio que profundice en las causas que motivan su elevado régimen pluviométrico.

La elevada pluviometría anual de la Sierra de Grazalema se atribuyó históricamente a su posición y orientación como primera barrera natural ante los frentes de procedencia atlántica que penetran en la península ibérica (Martín, 1991; Aparicio \& Silvestre, 1996; Capel, 2000; Gavira, 2006). Su orientación y altitud determina que los vientos procedentes del Atlántico, cargados de humedad, estén obligados a ascender por las laderas a barlovento, lo que provoca su condensación y descarga pluvial en dicha exposición. Por otra parte, a sotavento, no se recibe precipitación alguna, conociéndose este efecto como föhn, altamente estudiado en numerosas áreas de montaña y regiones polares (Bannister \& King, 2015; Nastos et al., 2017).

Como es bien conocido, el efecto föhn no produce lluvias per sé (Grosvenor et al., 2014; Nastos et al., 2017; van Drooge \& Ballesta, 2010). El término föhn se refiere estrictamente al ejemplo de los Alpes europeos, aunque tiene diferentes nombres en distintas regiones del mundo: en Málaga y Cádiz se denomina terral (aunque en Málaga sea de componente noroeste y en Cádiz de noreste). Normalmente, se caracteriza por ser un viento desecante que, en ningún caso, va asociado a lluvias (Monkhouse, 1978). Por otra parte, la «lluvia orográfica» tampoco explica las elevadas precipitaciones de la Sierra de Grazalema, puesto que los núcleos municipales de Grazalema y Villaluenga del Rosario, a sotavento de las sierras del Endrinal, Caíllo y Ubrique, registran valores pluviométricos más elevados que Ubrique y Benaocaz, núcleos a barlovento. López et al. (1989) analizan una situación meteorológica acontecida en la Sierra de Grazalema (9-12 febrero de 1985), estimando las características climáticas mediante un análisis sintético. Señalan que Grazalema, situada a sotavento, registra precipitaciones más cuantiosas que municipios ubicados a barlovento y relacionan este hecho con un efecto Bernoulli (efecto Venturi) provocado por el encauzamiento del viento en el Puerto del Boyar.
La Junta de Andalucía (2010) en el Informe de Medio Ambiente anual (elaborado desde 1987) analiza las variables climáticas en el ámbito andaluz. No obstante, para esta zona considera erróneamente los valores climáticos de una única estación como válidos para toda el área geográfica en que se ubica. Un ejemplo son las Sierras Subbéticas Occidentales, a las que se le asignan los valores climáticos de Grazalema. Así, esto confirma que la Sierra de Grazalema podría ser considerada como una de las localizaciones de la península más interesantes climatológicamente, y cuyo origen pluviométrico no está lo suficientemente estudiado.

Por lo tanto, en el presente estudio se pretende demostrar a través de un análisis de carácter mesoclimático qué tipo de factor incide sobre las características pluviométricas de la Sierra de Grazalema. Para alcanzar dicho objetivo, se han creado nuevos puntos extrapolados georreferenciados y con valores pluviométricos empleando gradientes y considerando el factor exposición (solana y umbría). Como hipótesis principal, se parte de la observación de que la posición de los núcleos de Grazalema y Villaluenga del Rosario, situados en la vertiente de sotavento, marcan registros pluviométricos más elevados que en los núcleos situados a barlovento, intuyéndose un inusual efecto meteorológico denominado «Depresión de Sotavento», relacionado probablemente con el llamado efecto Venturi; dicho efecto no solo acelera el viento, sino que, al reducir la presión atmosférica, incrementa la probabilidad de precipitación.

Luego, el objetivo principal será determinar científicamente las causas que motivan las elevadas precipitaciones en la Sierra de Grazalema.

\section{Materiales y métodos}

\section{1. Área de estudio}

El área de estudio, Sierra de Grazalema, está localizada dentro de los límites del Parque Natural homónimo. Este Parque Natural, ubicado en Andalucía entre las provincias de Cádiz y Málaga (Figura 1), cuenta con una superficie total de, aproximadamente, 53.400 ha. Se encuentra situado en su mayor extensión en la provincia de Cádiz (72\%), correspondiendo el resto del territorio a la provincia de Málaga (28\%) (Junta de Andalucía, 2006). La UNESCO concede el título de Reserva de la Biosfera a la Sierra de Grazalema, siendo junto al Parque Nacional de Ordesa y Monte Perdido (Pirineos), el primer espacio en España que logra este distintivo internacional. Entre otros reconocimientos internacionales, el Parque forma parte del Catálogo Nacional de Especies Amenazadas 

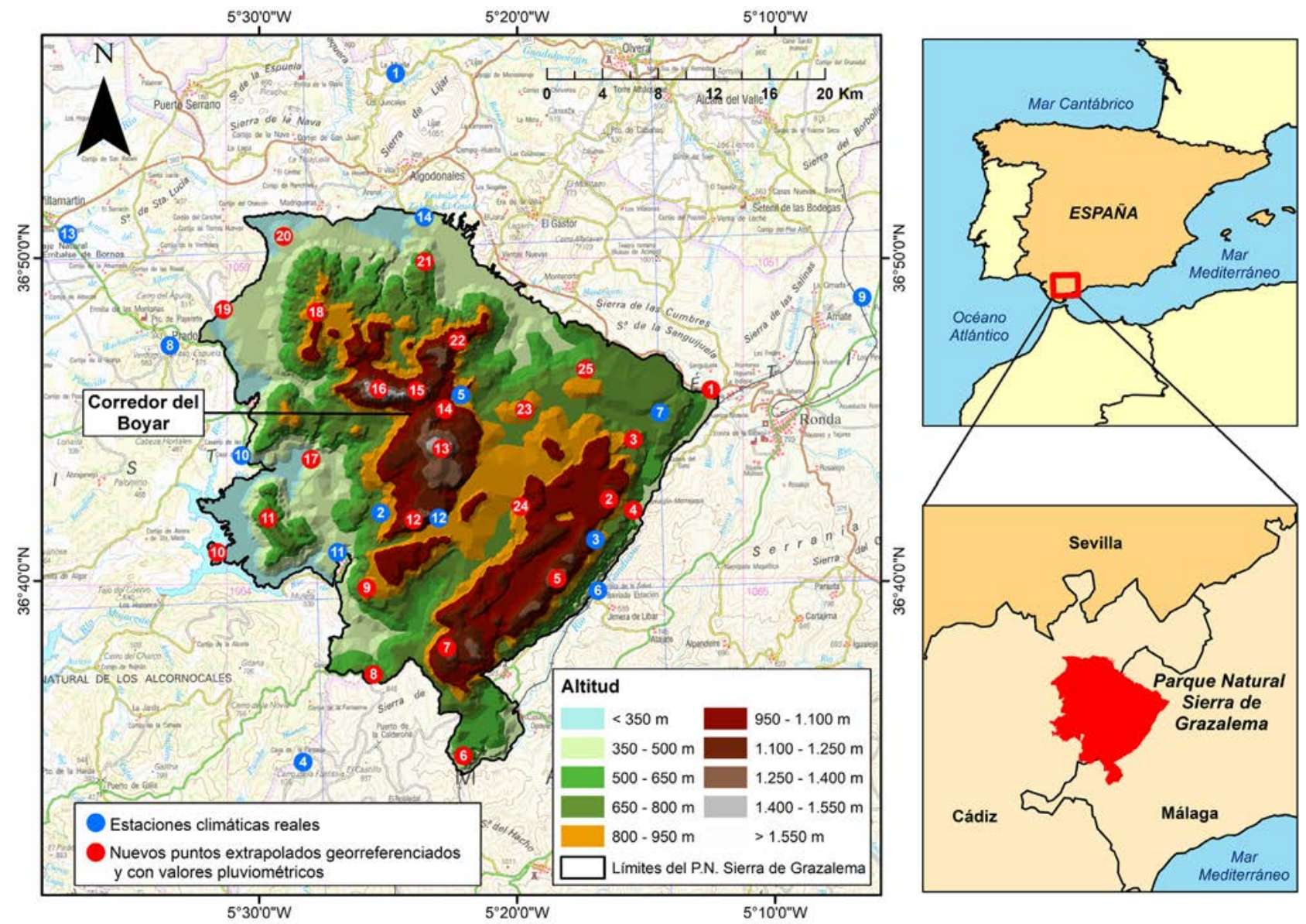

Figura 1: Localización del área de estudio. Fuente: elaboración propia a partir de datos del IECA.

Figure 1: Location of area of study. Source: based on the IECA.

(131 especies bajo la categoría de Especies de Interés Especial) y es Zona de Especial protección para las Aves, ZEPA (1979) (Junta de Andalucía, 2006).

Presenta una altitud media de 760 m s.n.m., localizándose su cota máxima en el pico del Pinar o Torreón (Sierra del Pinar) con 1.654 m s.n.m., que a su vez es la cota máxima de la provincia de Cádiz (Gavira, 2006).

La Sierra de Grazalema presenta un clima mediterráneo, caracterizado por un régimen pluviométrico con marcada estacionalidad, un período lluvioso invernal (precipitaciones medias en dicho período, en las estaciones analizadas, entre $200-870 \mathrm{~mm}$ ) y un verano largo y seco (Martín, 1991; Aparicio \& Silvestre, 1996). La cercanía al océano Atlántico $(80 \mathrm{~km}$.) y el efecto de agravación provocado por el relieve enmarca a esta zona en el dominio de la «España lluviosa» con precipitación media anual $>800 \mathrm{~mm}$ (Albentosa, 1989). Respecto al régimen térmico, presenta dos períodos claramente diferenciados: temperaturas medias elevadas en verano $\left(>20^{\circ} \mathrm{C}\right)$ y frescas durante el invierno $\left(<10^{\circ} \mathrm{C}\right)$. El relieve influye en su régimen térmico y en las especies vegetales que se pueden desarrollar, siendo evidente que las temperaturas disminuyen con la altitud (Aparicio \& Silvestre, 1996).
Según Pita (2003), la circulación atmosférica general sobre la Sierra de Grazalema destaca por la sucesión de centros de acción y de mecanismos meteorológicos diferentes en invierno y verano. En invierno es afectada por los vientos del oeste y perturbaciones del frente polar en capas bajas de la atmósfera, mientras que en verano el desplazamiento de los cinturones de bajas presiones hacia posiciones más septentrionales determina que quede bajo la influencia de las altas presiones subtropicales.

\subsection{Métodos}

\subsubsection{Organización de los datos climáticos}

Siguiendo los criterios espacio-temporales recomendados por la Organización Meteorológica Mundial (OMM), se han tomado datos de 14 estaciones (Tabla 1 y Figura 1) internas y periféricas a la zona objeto de estudio. Los datos utilizados provienen de las redes de observación del «Subsistema de información de climatología ambiental (CLIMA)» de la Red de Información Ambiental de Andalucía (REDIAM), aunque también se emplearon datos del «Sistema de información ambiental de An- 


\section{• • A. J. NARANJO-BAREA, J. RODRIGO-COMINO Y J. M. a SENCIALES-GONZÁLEZ}

Tabla 1: Índice general de las estaciones climáticas reales utilizadas en el estudio. Fuente: elaboración propia. Leyenda: ID: Código Estación; Pp: Precipitación; N: Número de años completos; X: Coordenada X (en metros); Y: Coordenada Y (en metros); Z: Altitud (en metros); Res. Temp.: Resolución temporal de los datos; AEMET: Agencia Estatal de Meteorología; AEMET ${ }^{1}$ : Red de Estaciones Secundarias de la Agencia Estatal de Meteorología; CHG: Confederación Hidrográfica del Guadalquivir; Hidrosur: Red de la Cuenca Mediterránea Andaluza; IFAPA: Instituto de Investigación y Formación Agraria y Pesquera.

Table 1: General index of the actual climatics stations used in the study. Source: own elaboration. Legend: ID:Station Code; Pp: Precipitation; N: Number of completed years; X: Coordinate X (in meters); Y: Coordinate Y (in meters); Z: Altitude (in meters); Res. Temp.: Temporal resolution of the data; AEMET: State Agency of Meteorology; AEMET': Network of Secondary Stations of the State Agency of Meteorology; CHG: Hydrographic Confederation of the Guadalquivir; Hidrosur: Network of the Andalusian Mediterranean Basin; IFAPA: Institute for Research and Training in Agriculture and Fisheries.

\begin{tabular}{|c|c|c|c|c|c|c|c|c|}
\hline \multirow{2}{*}{ Estación } & \multirow{2}{*}{ ID } & \multirow{2}{*}{ Res. Temp. } & \multirow{2}{*}{ Gestor } & \multirow{2}{*}{ Serie Pp } & \multirow{2}{*}{$\mathbf{N}$} & \multicolumn{3}{|c|}{ Coordenadas UTM 30S } \\
\hline & & & & & & $\mathbf{X}$ & $\mathbf{Y}$ & $\mathbf{Z}$ \\
\hline $\begin{array}{l}\text { Algodonales } \\
\text { (La Muela) }\end{array}$ & 5922 & Diaria y mensual & AEMET & $1968-2003$ & 25 & 285201.38 & 4089682.75 & 480 \\
\hline Benaocaz & 5944 & Diaria y mensual & $\mathrm{CHG}$ & $1968-2010$ & 15 & 283699 & 4064545.5 & 793 \\
\hline $\begin{array}{l}\text { Benaoján } \\
\text { (Cueva de la Pileta) }\end{array}$ & 6035 & Diaria y mensual & Hidrosur & $1961-2012$ & 30 & 296071.81 & 4062700 & 920 \\
\hline $\begin{array}{l}\text { Cortes de la Frontera } \\
\text { (Bañuelos) }\end{array}$ & 6039 & Diaria y mensual & Hidrosur & $1961-2010$ & 23 & 278862.38 & 4050327.5 & 560 \\
\hline $\begin{array}{l}\text { El Bosque (San José } \\
\text { Automática) }\end{array}$ & $5941 X$ & Diezminutal & AEMET & $2008-2015$ & 3 & 275763.2 & 4068008.92 & 297 \\
\hline Grazalema & E198 & Diezminutal & AEMET & $1991-2015$ & 12 & 288505.22 & 4071157.75 & 900 \\
\hline $\begin{array}{l}\text { Jimera de Líbar (Central } \\
\text { Eléctrica) }\end{array}$ & 6038 & Mensual & AEMET $^{1}$ & 1970-1994 & 16 & 296126.28 & 4059769 & 429 \\
\hline $\begin{array}{l}\text { Montejaque (Central } \\
\text { Eléctrica) }\end{array}$ & 6037 & Mensual & AEMET $^{1}$ & $1969-1995$ & 19 & 299964.72 & 4069855 & 530 \\
\hline Prado del Rey & 5928 & Diaria y mensual & CHG & $1967-2010$ & 28 & 271792.66 & 4074416.75 & 433 \\
\hline Ronda, Parchite & 6033 & Diaria & Hidrosur & 1961-2012 & 27 & 311767.66 & 4076216.5 & 755 \\
\hline Ubrique (Instituto) & 5945B & Diaria & AEMET & 1984-2006 & 13 & 281157.88 & 4062337.25 & 420 \\
\hline $\begin{array}{l}\text { Villaluenga del Rosario } \\
\text { (ICONA) }\end{array}$ & 5943B & Diaria & AEMET & $1984-2015$ & 13 & 287051.84 & 4064157.73 & 885 \\
\hline Villamartín & RIA1104 & Diaria & IFAPA & $2001-2015$ & 10 & 266087.6 & 4080966.64 & 151 \\
\hline Zahara de la Sierra (Presa) & $5912 \mathrm{C}$ & Diaria & $\mathrm{CHG}$ & $1992-2015$ & 11 & 286633.72 & 4081397.47 & 393 \\
\hline
\end{tabular}

dalucía (SINAMBA)» (Junta de Andalucía, 1996) para subsanar lagunas en varias series de precipitación; debemos puntualizar que, aunque los datos de las estaciones han sido extraídos de REDIAM y SINAMBA, la fuente original de los datos es: Agencia Estatal de Meteorología (AEMET), Confederación Hidrográfica del Guadalquivir (CHG), Red de la Cuenca Mediterránea Andaluza (Hidrosur) e Instituto de Investigación y Formación Agraria y Pesquera (IFAPA).

Previamente al análisis pluviométrico, fue necesario ordenar los datos a la misma escala temporal, pues los datos en bruto no eran comparables: diezminutales, diarios y mensuales. Este proceso, además, permitió hallar lagunas en series y establecer los siguientes criterios para su aceptación o rechazo:

- En series pluviométricas diezminutales pueden faltar, por un lado, un máximo de 10 tramos horarios y, por el otro, un máximo de 5 días.
- En series de precipitación diarias, puede faltar un máximo de 2 días.

- Carecer de un mes supone suprimir ese año.

Se dispuso de datos pluviométricos de 14 estaciones, de las cuales se eliminó la de «El Bosque, San José Automática», ya que solo cuenta con 3 años de observación completos. El período básico de trabajo fue 1961-2015 (Tabla 1).

\subsubsection{Análisis detallado de precipitaciones}

Teniendo los datos en cada una de las estaciones clasificados en volúmenes mensuales, hemos creído oportuno realizar un breve análisis estacional; para evitar posibles ambigüedades, se ha considerado adecuado citar los meses que, en el hemisferio norte, conforman las 4 estaciones del año (clasificación tomada de Albentosa, 1990): 
- Invierno: diciembre, enero y febrero.

- Primavera: marzo, abril y mayo.

- Verano: junio, julio y agosto.

- Otoño: septiembre, octubre y noviembre.

Para analizar su validez estadística se calcularon las siguientes medidas de centralidad y dispersión: promedios y desviaciones estándar, coeficientes de variación, sesgo (coeficiente de asimetría de Fisher) y medianas (Material suplementario 1). También se cuantificaron el número medio de días de precipitación (promedio del sumatorio de días de precipitación mensual, $\mathrm{n}^{\circ}$ de días) y la intensidad media (dividiendo el volumen pluviométrico medio mensual por el número medio mensual de días de precipitación, $\mathrm{mm} /$ día) que fue clasificada según Senciales (1995)

Además, se han analizado todos los eventos pluviométricos que se han registrado en la estación de «Grazalema» en el período 1992-2015, para valorar la intensidad de estos eventos. Se ha hecho especial hincapié en analizar los eventos torrenciales (aquellos que superaron los $100 \mathrm{~mm} / 24 \mathrm{~h}$ ). Así mismo, se ha calculado (a través del modelo probabilístico de Poisson: $\mathrm{P}=\mathrm{e}^{-\lambda} * \lambda^{\mathrm{x}} / \mathrm{x}$ !: Senciales \& Ruiz-Sinoga, 2013) la probabilidad de recurrencia de estos eventos. También, tomando los datos de los archivos históricos de Wetterzentrale (2017) se han clasificado estos eventos torrenciales ( $\mathrm{pp}>100-150 \mathrm{~mm} / 24 \mathrm{~h}$ ) en función de la dinámica atmosférica, es decir, se ha realizado una interpretación de la situación sinóptica que determinó la lluvia torrencial.

\subsubsection{Estimación de nuevos puntos pluviométricos}

Con la información recopilada pueden analizarse en detalle las precipitaciones; sin embargo, con objeto de aumentar la precisión del análisis, se crearon también nuevos puntos pluviométricos dentro del área de estudio, seleccionando diversas cotas topográficas con el objetivo de cubrir la totalidad del área estudiada, para obtener una distribución homogénea en la que no quedasen grandes zonas de ausencia de datos. A continuación, se procedió a su extrapolación, basada en gradientes, desde los valores de las estaciones reales.

Para obtener los nuevos puntos se seleccionaron, introduciendo las «Hojas 1036-3, 1049-2 y 4, 1050-1 a 4, y 1064-1 a 4 del Mapa Topográfico Nacional de España, escala 1:25.000 ráster» (IGN) en el programa ArcMap 10.5 (ESRI, 2016), 25 puntos (cotas topográficas) internos al área de estudio. Se trató que la distribución de estos puntos fuera regular, sin grandes zonas de ausencia de datos, y su densidad relativamente alta (Figura 1).

La exposición es el segundo condicionante, pues no es válido un mismo gradiente pluviométrico para zonas con distinta exposición. Aquí se optó por clasificar las estaciones climáticas reales y los nuevos puntos pluviométricos según su disposición en solana (sur) o umbría (norte), utilizando el mapa de orientaciones de la zona objeto de estudio (Figura 2) y «el Servicio WMS (Web Map Servi- ce) que dispone de un mapa de orientaciones de $100 \times 100 \mathrm{~m}$. de Andalucía (para escalas menores a 1:200.000)» (REDIAM, 2006), agrupando en solana las estaciones con orientación sur, suroeste y sureste, y en umbría las de orientación norte, noroeste y noreste (Tabla 2). Cabe destacar que se utilizaron los términos solana-umbría para calcular los gradientes y no barloventosotavento porque tras evaluar los datos con un análisis ANOVA (SPSS 23.0 [IBM, 2014]), no existió relación alguna entre precipitaciones y exposición (a causa, como veremos del efecto «depresión de sotavento»).

Para el cálculo de los gradientes pluviométricos anuales se halló, mediante estimaciones lineales (gradientes), su validez (coeficiente de correlación) y el punto de inicio de la recta desde el cual es calculable cualquier punto de la Sierra de Grazalema (intersección del eje). El coeficiente de correlación $\left(\mathrm{R}^{2}\right)$ señala un valor positivo de correlación (valores muy bajos llevan a depurar estaciones que producen error), y la intersección del eje (I) señala el punto de inicio de cualquier cálculo, bajándolo a un teórico nivel de 0 m (Aguiló, 2004). De esta forma, se consideraron para solana: «Grazalema», «Prado del Rey», «Ubrique, Instituto» $\mathrm{y}$ «Villaluenga del Rosario, Icona» con un ajuste lineal de $\mathrm{R}^{2}=0.87$. Para umbría: «Benaocaz», «Jimera de Líbar, Central Eléctrica», «Villamartín» y «Zahara de la Sierra, Presa» con un ajuste lineal de $\mathrm{R}^{2}=0.91$.

Así, pese a disponer de menos estaciones asociadas de las que se partía en principio, se obtuvo, en gran medida, un gradiente ajustado a la realidad y, por tanto, pueden considerarse gradientes con una elevada correlación (tendencia a asemejarse a una ecuación lineal).

Conociendo altitud y exposición de los 25 nuevos puntos extrapolados georreferenciados y con valores pluviométricos (Figura 1 y Tabla 2), solo se deben calcular, para cada una, sus valores pluviométricos (precipitación media anual). Esta estimación se efectuó utilizando los gradientes válidos (para solana y umbría) obtenidos anteriormente, mediante la fórmula de extrapolación propuesta por Aguiló (2004):

$$
\text { Pp }(\text { a una altura } h)=I+\left(\gamma^{*} h\right)
$$

Donde:

$\mathrm{Pp}=$ Precipitación.

$\mathrm{I}=$ Punto de intersección calculado.

$\mathrm{\gamma}=$ Gradiente.

$\mathrm{h}=$ Altura del punto que se quiere calcular.

Tras los cálculos, se consigue crear nuevos puntos extrapolados georreferenciados y con valores pluviométricos que permiten, junto a las estaciones climáticas reales, obtener una cartografía pluviométrica.

\subsubsection{Cartografía pluviométrica}

Creados los 25 puntos extrapolados georreferenciados y con valores pluviométricos, junto con las estaciones 


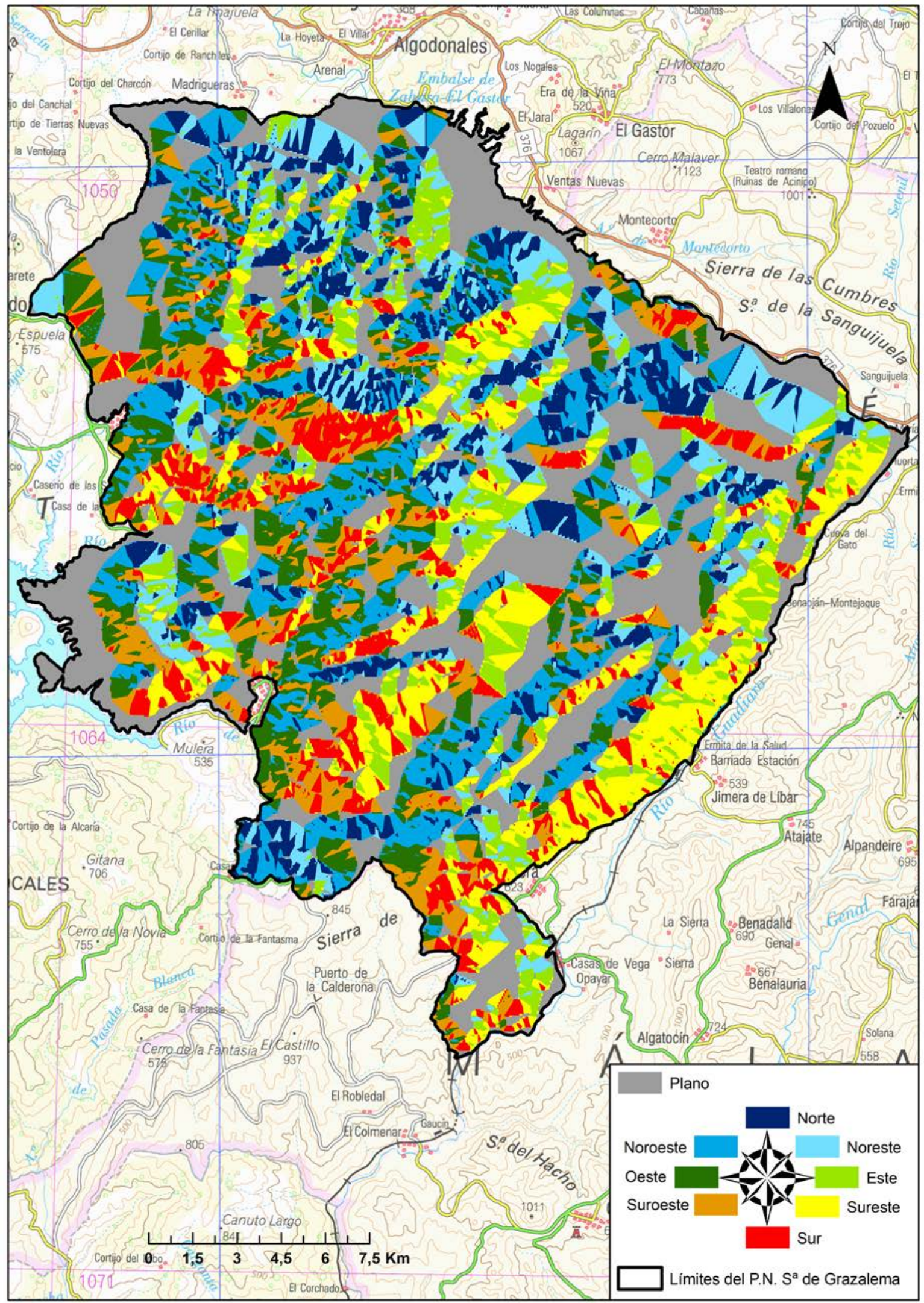

Figura 2: Mapa de orientaciones del área de estudio. Fuente: Elaboración propia a partir de datos del IECA e IGN (2011). Figure 2: Map of orientations of the study area. Source: based on the IECA and IGN (2011). 
Tabla 2. Exposición de las estaciones climáticas reales y de los nuevos puntos extrapolados. Elaboración propia. Leyenda: $\left(\mathrm{n}^{\circ}\right)$ : Se corresponde con la Figura 1.

Table 2. Exposition of the real stations and the new extrapolated points. Source: own elaboration. Legend: $\left(n^{\circ}\right):$ Corresponds to Figure 1.

\begin{tabular}{|c|c|c|}
\hline \multirow{2}{*}{ Estaciones climáticas } & \multicolumn{2}{|c|}{ Exposición } \\
\hline & Solana & Umbría \\
\hline Reales & $\begin{array}{l}\text { (3) Benaoján (Cueva de la Pileta); (4) Cortes } \\
\text { de la Frontera (Bañuelos); (5) Grazalema; } \\
\text { (8) Prado del Rey; (9) Ronda (Parchite); } \\
\text { (11) Ubrique (Instituto); (12) Villaluenga del } \\
\text { Rosario (ICONA). }\end{array}$ & $\begin{array}{l}\text { (1) Algodonales (La Muela); (2) Benaocaz; (10) } \\
\text { El Bosque (San José, Automática); (6) Jimera de } \\
\text { Líbar (Central Eléctrica); (7) Montejaque (Central } \\
\text { Eléctrica); (13) Villamartín; (14) Zahara de la } \\
\text { Sierra (Presa). }\end{array}$ \\
\hline Nuevos puntos extrapolados & $\begin{array}{l}\text { (1) Cerro de Cueva Bermeja; (2) Pico } \\
\text { Ventana; (3) Cerro Hacho; (4) Pico Tajo } \\
\text { de la Dehesa; (5) Pico Palo; (6) Pico } \\
\text { Pajarraquera; (14) Pico Peñón Grande; (22) } \\
\text { Pico Coros; (24) Pico La Breña. }\end{array}$ & $\begin{array}{l}\text { (7) Sierra de los Pinos; (8) Peñón del Berrueco; (9) } \\
\text { Cerro del Algarrobal; (10) Cerro de la Caldera; (11) } \\
\text { Pico Silla; (12) Pico Navazo Alto; (13) Cerro del } \\
\text { Simancón; (15) Pico San Cristóbal; (16) Pico Pinar; } \\
\text { (17) Castillo Aznamara; (18) Sierra Margarita; } \\
\text { (19) Cerro del Conejo; (20) Puerto de Zaragute; } \\
\text { (21) Pico Pulgón; (23) Pico Jaral; (25) Cerro del } \\
\text { Carrascal. }\end{array}$ \\
\hline
\end{tabular}

reales seleccionadas, se logra una alta densidad de información en el área de estudio (Figura 1), permitiendo elaborar una cartografía climática detallada y, posteriormente, valorar la incidencia en profundidad del relieve sobre las características climáticas de la Sierra de Grazalema. La cartografía detallada se restringió a la precipitación media anual, aunque es posible elaborar también para análisis más profundos una cartografía mensual.

Son numerosos los autores que, ante la exigua concentración de estaciones climáticas, han optado por la utilización de diversos métodos de interpolación para la realización de la cartografía climática (Daly et al., 1994; Atkinson \& Lloyd, 1998; Goovaerts, 2000; Vicente-Serrano \& SazSánchez, 2002). En las últimas décadas, en el ámbito científico, ha surgido un debate acerca de cuál es el mejor método de interpolación para producir la cartografía climática (Vicente-Serrano \& Saz-Sánchez, 2002); fruto de este debate han surgido numerosos estudios en los cuales se han analizado y comparado multitud de métodos de interpolación de la precipitación y la temperatura en diferentes regiones del mundo (Tabios III \& Salas, 1985; Kurtzman \& Kadmon, 1999; Zimmerman et al., 1999; Bustamante, 2003; Izquierdo \& Márquez, 2007; Keblouti et al., 2012).

Ante esto, y con la intención de realizar comparación de los resultados obtenidos en los distintos métodos de interpolación, hemos optado por realizar la misma cartografía pluviométrica con IDW (Inverse Distance Weighted) con el programa ArcMap 10.5 (ESRI, USA) a una escala de 1:150.000. Hay que señalar que para la obtención del mapa por el método IDW se han utilizado únicamente los datos de las estaciones climáticas reales y los nuevos puntos pluviométricos.

\subsubsection{Cálculo efecto Venturi}

Para corroborar la hipótesis y siguiendo las premisas de Martín (1991), se estimó el cálculo del efecto Venturi comparando presión y velocidad del aire entrante en el Corredor del Boyar ( $36^{\circ} 45^{\prime} 21^{\prime \prime} \mathrm{N}, 5^{\circ} 23^{\prime} 42^{\prime \prime} \mathrm{W}$ ) con los mismos parámetros del aire saliente en Grazalema. Para ello, en primer lugar, se cubicó el volumen del aire entrante por el Puerto del Boyar (Figura 3a), y el saliente por Grazalema helipuerto (Figura 3b), Grazalema estación (Figura 3c) y Grazalema ayuntamiento (Figura 3d). Y, por último, a partir de la ecuación de Bernoulli (Mott, 2006), se estimaron los cambios de presión (efecto Venturi) entre dichos puntos (Tabla 3 ):

$$
\frac{\mathrm{Pl}}{\gamma}+\mathrm{zl}+\frac{\mathrm{v} 1^{2}}{2 \mathrm{~g}}=\frac{\mathrm{P} 2}{\gamma}+\mathrm{z} 2+\frac{\mathrm{v} 2^{2}}{2 \mathrm{~g}}
$$

Donde:

$\mathrm{P}=$ Presión atmosférica $(\mathrm{KPa})$.

$\gamma=$ Densidad del aire $\left(\mathrm{Kg} / \mathrm{m}^{3}\right)$.

$\mathrm{Z}=$ Altura de la masa $(\mathrm{Hm})$.

$\mathrm{v}=$ Velocidad del viento $(\mathrm{m} / \mathrm{s})$.

$\mathrm{g}=\operatorname{Gravedad}\left(\mathrm{m} / \mathrm{s}^{2}\right)$.

Existen, en el interior de la Sierra de Grazalema, dos corredores (Manga de Villaluenga [36 $36^{\circ} 41^{\prime} 15^{\prime \prime} \mathrm{N}, 5^{\circ} 24^{\prime}$ 31 " W] y Pasillo de Líbar [36 41'3" N, 5 $5^{\circ} 18$ ' 25" W]) con una configuración similar a la del Corredor del Boyar. Esto puede dar lugar a canalizaciones del aire que provoquen efectos Venturi. Es por ello, por lo que en dichos corredores se llevaron a cabo los mismos cálculos y estimaciones descritos anteriormente para el Corredor del Boyar, con la intención de estimar los cambios de presión (Tabla 3) entre los distintos puntos de la Manga de Villaluenga (Figuras 3e, 4a y 4b), y el Pasillo de Líbar (Figu$\operatorname{ras} 4 \mathrm{c}, 4 \mathrm{~d}$ y $4 \mathrm{e})$.

Hay que señalar que para dichos cálculos (Tabla 3) se parte de una velocidad inicial del viento de $3 \mathrm{~m} / \mathrm{s}$; una presión inicial semejante para toda el área de montaña, 


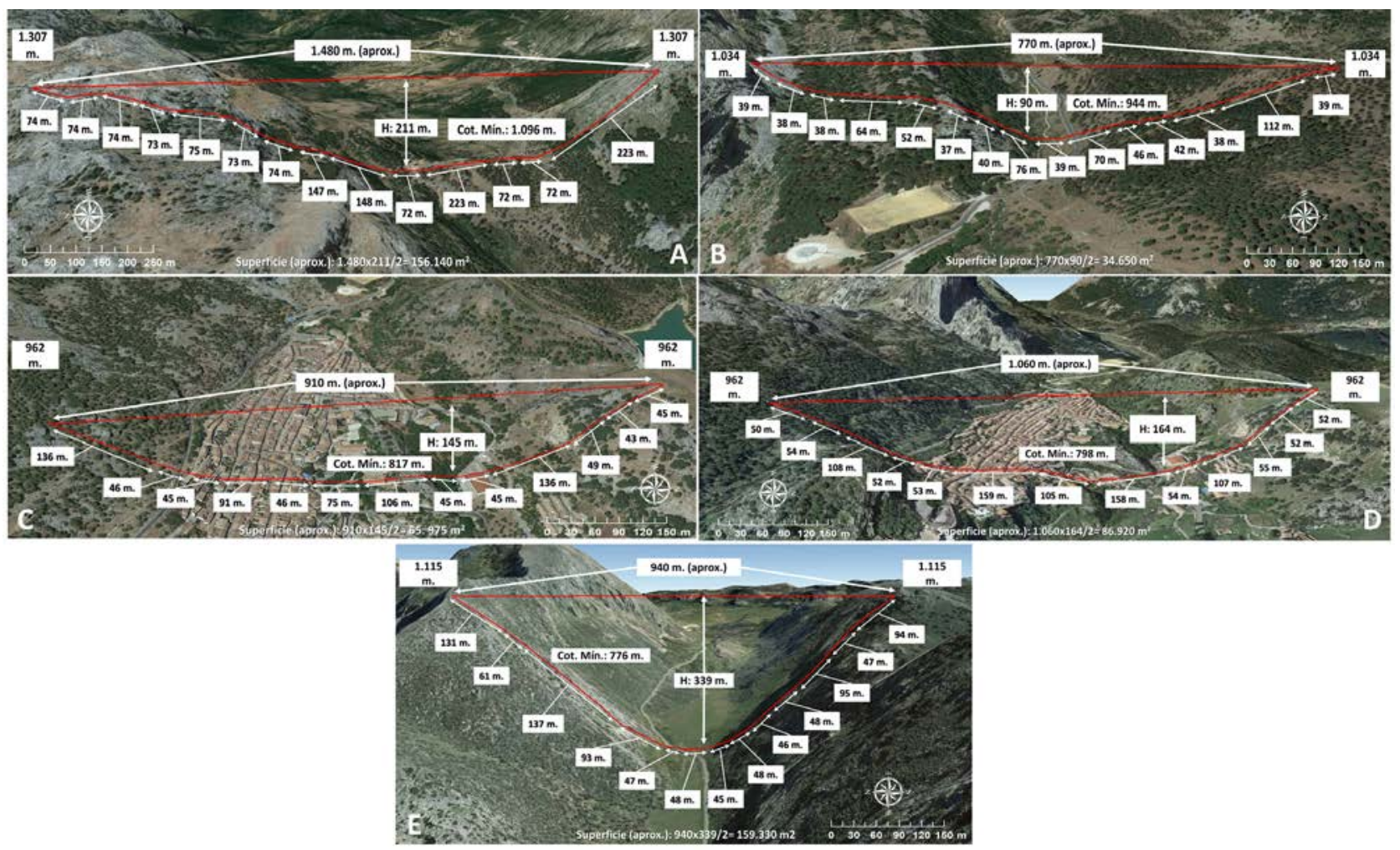

Figura 3: Mediciones en el Puerto del Boyar (A), Grazalema helipuerto (B), Grazalema estación (C), Grazalema ayuntamiento (D) y Boca Villaluenga (E). Fuente: elaboración propia a partir de imagen satélite de Google Earth (2017).

Figure 3: Measurements in the Puerto del Boyar (A), Grazalema helipuerto (B), Grazalema estación (C), Grazalema ayuntamiento (D) and Boca Villaluenga (E). Source: based on the satellite image of Google Earth (2017).

Tabla 3: Cálculos efecto Venturi en la Sierra de Grazalema. Fuente: elaboración propia. Leyenda: (*) Hmasa 1 y 2: Altura de la lámina de aire entre fondo de valle y cumbre antes de desbordar. Res. V2 (final)= Resultado de la velocidad final respecto a la inicial; Res. P2 (final)= Resultado de la presión final empleando la fórmula de Bernoulli.

Table 3: Calculations Venturi effect in the Grazalema Mountain. Source: own elaboration. Legend: Hmasa 1 y 2: Height of the air sheet between bottom of valley and summit before overflowing. Res. V2 (final)= Result of the final velocity with respect to the initial velocity; Res. P2 (final)= Result of the final pressure using Bernoulli's formula.

\begin{tabular}{|l|c|c|c|c|c|c|c|c|c|}
\hline \multicolumn{1}{|c|}{ Estación } & $\begin{array}{c}\text { Sección 1 } \\
\left(\mathbf{m}^{\mathbf{2}} \mathbf{n}\right.\end{array}$ & $\begin{array}{c}\text { Sección } \mathbf{2} \\
\left(\mathbf{m}^{\mathbf{2}}\right)\end{array}$ & $\begin{array}{c}\text { Hmasa 1 } \\
(*)\end{array}$ & $\begin{array}{c}\text { Hmasa 2 } \\
(*)\end{array}$ & $\begin{array}{c}\text { Res. V2 } \\
(\mathbf{f i n a l})\end{array}$ & $\begin{array}{c}\text { Res. P2 } \\
(\mathbf{f i n a l})\end{array}$ & $\begin{array}{c}\text { Vol. Precipitable } \\
\mathbf{2}\end{array}$ & $\begin{array}{c}\text { Dif. } \\
\mathbf{1 - 2}\end{array}$ & $\begin{array}{c}\text { Incremento } \\
\text { (\%) }\end{array}$ \\
\hline $\begin{array}{l}\text { Pto. Boyar-Grazalema } \\
\text { helipuerto }\end{array}$ & 156.140 & 34.650 & 211 & 367 & 13.52 & 77.33 & 2.42 & 142.98 & 42.98 \\
\hline $\begin{array}{l}\text { Pto. Boyar-Grazalema } \\
\text { estación }\end{array}$ & 156.140 & 65.975 & 211 & 145 & 7.10 & 87.20 & 1.79 & 105.99 & 5.99 \\
\hline $\begin{array}{l}\text { Pto. Boyar-Grazalema } \\
\text { ayuntamiento }\end{array}$ & 156.140 & 86.920 & 211 & 164 & 5.39 & 88.19 & 1.73 & 102.28 & 2.28 \\
\hline $\begin{array}{l}\text { Boca Villaluenga- } \\
\text { Villaluenga estación }\end{array}$ & 159.330 & 128.997 & 339 & 198 & 3.71 & 90.09 & 1.61 & 95.18 & -4.82 \\
\hline $\begin{array}{l}\text { Pto. Villaluenga- } \\
\text { Villaluenga estación }\end{array}$ & 136.620 & 128.997 & 253 & 198 & 3.18 & 89.34 & 1.66 & 97.96 & -2.04 \\
\hline $\begin{array}{l}\text { Boca Villaluenga-Pto. } \\
\text { Villaluenga }\end{array}$ & 159.330 & 136.620 & 339 & 253 & 3.5 & 89.56 & 1.64 & 97.14 & -2.86 \\
\hline $\begin{array}{l}\text { Cortijo Líbar-Fuente } \\
\text { Cufría }\end{array}$ & 225.120 & 198.170 & 268 & 266 & 3.41 & 88.68 & 1.70 & 100.47 & 0.47 \\
\hline $\begin{array}{l}\text { Cortijo Líbar-Ent. } \\
\text { Montejaque }\end{array}$ & 225.120 & 162.402 & 268 & 263 & 4.16 & 88.39 & 1.72 & 101.54 & 1.54 \\
\hline
\end{tabular}




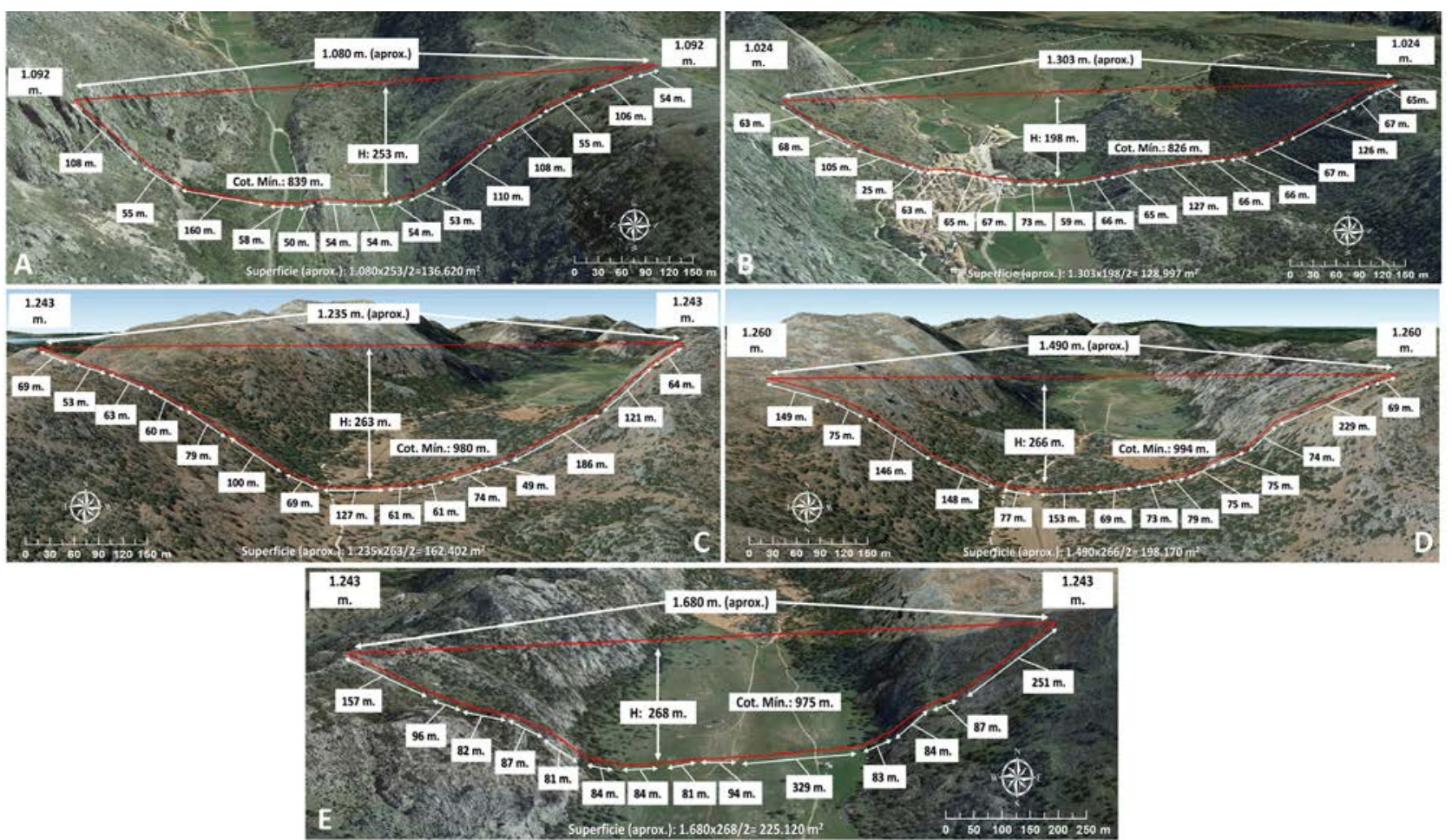

Figura 4: Mediciones en el Puerto de Villaluenga (A), Villaluenga estación (B), Entrada Pasillo de Líbar desde Montejaque (C), Fuente de Cufría (D) y Cortijo de Líbar (E). Fuente: elaboración propia a partir de imagen satélite de Google Earth (2017). Figure 4: Measurements in the Puerto de Villaluenga (A), Villaluenga estación (B), Entrada Pasillo de Libar desde Montejaque (C). Fuente de Cufria (D) y Cortijo de Libar (E). Source: based on the satellite image of Google Earth (2017).

estimada en $88.8 \mathrm{KPa}$; una densidad media de $1.1 \mathrm{Kg} / \mathrm{m}^{3}$ de aire; y el valor estándar de la gravedad $\left(9.8 \mathrm{~m} / \mathrm{s}^{2}\right)$, todo ello aplicado a la expresión de Bernoulli (Mott, 2006). Debemos apuntar, también, que se parte de un valor estándar de presión de $62.12 \mathrm{KPa}$ a $4.000 \mathrm{~m}$; un volumen de agua precipitable $(\mathrm{w})$ estandarizado a 0.62 (volumen de masa húmeda/volumen de masa seca); y un volumen precipitable (calculable mediante la siguiente expresión: pw $\left.=\mathrm{W}^{*}(\mathrm{p} 1-\mathrm{p} 2) / 9.8\right)$ estimado en $1.69 \mathrm{~mm} / \mathrm{m}^{3}$ de aire.

\section{Resultados}

\subsection{Análisis detallado de las precipitaciones}

Las precipitaciones medias anuales oscilan entre 584 y $1.893 \mathrm{~mm}$ (material suplementario 1), correspondiendo el valor más bajo a «Montejaque, Central Eléctrica» (429 m) y el más elevado a «Grazalema» (900 m). Estos datos permiten incluir a la Sierra de Grazalema y su área adyacente en una región de clima húmedo (salvo «Montejaque, Central Eléctrica»), puesto que todas las estaciones tienen precipitaciones medias anuales que oscilan entre el ombroclima subhúmedo y el hiperhúmedo (Rivas, 1983).

Es problemática la definición de barlovento-sotavento en la Sierra de Grazalema. En general, se aprecia mayor pluviometría en la vertiente de barlovento que en la de sotavento, como parece lógico; sin embargo, existen unas «islas» pluviométricas marcadas por «Grazalema» $\mathrm{y}$ «Villaluenga del Rosario, Icona» que rompen dicha lógica ya que registran precipitaciones medias anuales superiores a las estaciones ubicadas en la vertiente de barlovento.

Los registros pluviométricos medios anuales de las estaciones situadas en ambas vertientes (dicha comparación se efectúa entre estaciones que ocupan una posición latitudinal similar): al norte, en barlovento, «Villamartín» a $151 \mathrm{~m}$ presenta precipitaciones medias anuales de 615 mm, y «Prado del Rey» a 433 m, 789 mm; en cambio, a sotavento, las precipitaciones se reducen considerablemente: «Montejaque, Central Eléctrica», a $530 \mathrm{~m}$, registra $584 \mathrm{~mm}$; «Zahara de la Sierra, Presa», a 393 m, 686 mm; «Algodonales, La Muela», a $480 \mathrm{~m}, 702 \mathrm{~mm}, \mathrm{y}$ «Ronda, Parchite», a $755 \mathrm{~m}$ solo recibe $643 \mathrm{~mm}$.

Descendiendo en latitud, las diferencias pluviométricas medias anuales entre ambas vertientes se acentúan: barlovento alberga estaciones con registros pluviométricos superiores, pese a que estas se ubican a menor altitud que sotavento; en dicha vertiente, «Ubrique, Instituto», a $420 \mathrm{~m}$, recibe $1.015 \mathrm{~mm}$, y «Benaocaz», a $793 \mathrm{~m}, 1.416$ $\mathrm{mm}$. Contrariamente, a sotavento, «Jimera de Líbar, Central Eléctrica», a 429 m, presenta 934 mm, y «Benaoján, Cueva de la Pileta», a $920 \mathrm{~m}$ registra $1.322 \mathrm{~mm}$.

Mención aparte merecen, al registrar los valores pluviométricos medios anuales más elevados del área objeto de estudio, «Cortes de la Frontera, Bañuelos», «Villaluenga del Rosario, Icona» y «Grazalema». En barloven- 
Material suplementario 1: Medidas de centralidad y dispersión de las precipitaciones de las 13 estaciones climáticas utilizadas en el análisis pluviométrico. Fuente: elaboración propia. Leyenda: Desv. Est.= desviación estándar; Coef. Var.= coeficiente de variación; en cada estación se señala en azul el valor pluviométrico medio mensual más bajo y en rojo el más elevado.

\begin{tabular}{|c|c|c|c|c|c|c|c|c|c|c|c|c|c|c|c|}
\hline Estación & $\begin{array}{c}\mathrm{N}^{0} \text { años } \\
\text { completos }\end{array}$ & $\begin{array}{l}\text { Estimador } \\
\text { estadístico }\end{array}$ & E & F & M & A & My & $\mathbf{J}$ & $\mathrm{Jl}$ & A & S & 0 & $\mathbf{N}$ & D & Año \\
\hline \multirow{5}{*}{$\begin{array}{l}\text { Algodonales } \\
\text { (La Muela) }\end{array}$} & \multirow{5}{*}{25} & Media & 107.5 & 76.2 & 71.1 & 58.9 & 46.7 & 16.9 & 1.1 & 7.6 & 31 & 66.3 & 86.1 & 132.6 & 702 \\
\hline & & Mediana & 95.5 & 65 & 66 & 37 & 32 & 7 & 0 & 0 & 23 & 35.8 & 55 & 99 & 713 \\
\hline & & Desv. Est. & 87.5 & 73.3 & 52.5 & 52.4 & 41 & 23.5 & 5 & 16.7 & 33.5 & 73.4 & 94.1 & 111.4 & 178.1 \\
\hline & & Coef. Var. & 81.4 & 96.2 & 73.8 & 89 & 87.7 & 138.8 & 462.9 & 219.5 & 108.3 & 110.7 & 109.3 & 84 & 25.4 \\
\hline & & Sesgo & 1.2 & 1.3 & 0.8 & 1 & 0.8 & 1.7 & 5 & 2.6 & 1.3 & 1.1 & 1.6 & 0.9 & 0.2 \\
\hline \multirow{5}{*}{ Benaocaz } & \multirow{5}{*}{15} & Media & 241.3 & 194.3 & 157.2 & 122.9 & 95.5 & 49.5 & 2.8 & 11.6 & 22.5 & 127.5 & 183.6 & 207 & 1.416 \\
\hline & & Mediana & 151 & 89 & 146 & 118 & 68 & 29 & 0 & 0 & 11 & 91 & 136 & 168 & 1.288 \\
\hline & & Desv. Est. & 265.9 & 174.1 & 114.8 & 77.8 & 87.2 & 55.9 & 9.5 & 32.2 & 34.6 & 122.4 & 154.4 & 158.6 & 384.1 \\
\hline & & Coef. Var. & 110.2 & 89.6 & 73 & 63.3 & 91.3 & 112.9 & 335.7 & 277.3 & 153.7 & 95.9 & 84.1 & 76.6 & 27.1 \\
\hline & & Sesgo & 1.6 & 0.8 & 1.2 & 0.8 & 0.7 & 1.5 & 3.8 & 3.5 & 2.4 & 1.4 & 1.5 & 0.7 & 0.4 \\
\hline \multirow{5}{*}{$\begin{array}{l}\text { Benaoján } \\
\text { (Cueva de la } \\
\text { Pileta) }\end{array}$} & \multirow{5}{*}{30} & Media & 210.4 & 180.9 & 120.6 & 103.1 & 74.7 & 18.9 & 2.2 & 6.9 & 35.8 & 144.4 & 192.3 & 232.1 & 1.322 \\
\hline & & Mediana & 151.7 & 156.8 & 113.2 & 87.1 & 51.6 & 5.2 & 0 & 0.3 & 17.7 & 126.8 & 169.9 & 130.1 & 1.252 \\
\hline & & Desv. Est. & 185.7 & 151.1 & 104.3 & 90.4 & 71.8 & 27.5 & 6.7 & 18.7 & 43.1 & 124 & 162.9 & 241 & 487.6 \\
\hline & & Coef. Var. & 88.2 & 83.6 & 86.5 & 87.7 & 96.1 & 145.5 & 302.1 & 270.1 & 120.4 & 85.9 & 84.7 & 103.8 & 36.9 \\
\hline & & Sesgo & 0.9 & 0.9 & 1.3 & 2.1 & 1.2 & 1.6 & 4.5 & 4.3 & 1.3 & 0.8 & 1.2 & 1.6 & 0.8 \\
\hline \multirow{5}{*}{$\begin{array}{l}\text { Cortes de } \\
\text { la Frontera } \\
\text { (Bañuelos) }\end{array}$} & \multirow{5}{*}{23} & Media & 249.7 & 223.6 & 153.5 & 134.1 & 95.6 & 27.6 & 6.4 & 6.6 & 51.5 & 219.9 & 253.2 & 303.3 & 1.725 \\
\hline & & Mediana & 174 & 134 & 123 & 93 & 84 & 9 & 0 & 3 & 22 & 123 & 168.4 & 200.7 & 1.525 \\
\hline & & Desv. Est. & 210.4 & 183.3 & 124.5 & 106.4 & 90.1 & 39.3 & 18.1 & 10.3 & 72.7 & 227.2 & 223.9 & 332.7 & 647.8 \\
\hline & & Coef. Var. & 84.3 & 82 & 81.1 & 79.3 & 94.2 & 142.3 & 283 & 155.7 & 141.1 & 103.3 & 88.4 & 109.7 & 37.6 \\
\hline & & Sesgo & 1.3 & 0.9 & 0.7 & 0.8 & 1.2 & 1.8 & 3.4 & 1.9 & 2.2 & 1.8 & 1.2 & 1.5 & 0.9 \\
\hline \multirow{5}{*}{ Grazalema } & \multirow{5}{*}{12} & Media & 329.6 & 187 & 228.6 & 186.4 & 99.9 & 19.7 & 0.4 & 4.5 & 80.8 & 166.9 & 238 & 351.2 & 1.893 \\
\hline & & Mediana & 203.5 & 152 & 140.9 & 121.8 & 72.7 & 5.9 & 0 & 0.8 & 73.4 & 164.4 & 170.4 & 274.7 & 1.914 \\
\hline & & Desv. Est. & 306.7 & 172.5 & 279.4 & 178.7 & 86.2 & 42.1 & 0.7 & 8.7 & 53.5 & 86.3 & 199.1 & 341.7 & 747.7 \\
\hline & & Coef. Var. & 93 & 92.3 & 122.2 & 95.9 & 86.3 & 214.1 & 166.9 & 190.7 & 66.1 & 51.7 & 83.7 & 97.3 & 39.5 \\
\hline & & Sesgo & 0.7 & 1.4 & 2.6 & 1.3 & 2.4 & 3.2 & 1.5 & 2.9 & 0.9 & -0.1 & 0.8 & 1.7 & 0.2 \\
\hline \multirow{5}{*}{$\begin{array}{l}\text { Jimera } \\
\text { de Líbar } \\
\text { (Central } \\
\text { Eléctrica) }\end{array}$} & \multirow{5}{*}{16} & Media & 141.1 & 117.1 & 96.7 & 74.1 & 68.1 & 23.3 & 1.4 & 3.3 & 15.6 & 71.8 & 158.4 & 162.8 & 934 \\
\hline & & Mediana & 105 & 103.5 & 94 & 70.5 & 69 & 10 & 0 & 1 & 5.5 & 35 & 102.5 & 179.5 & 878 \\
\hline & & Desv. Est. & 167.9 & 86.5 & 78.9 & 56.8 & 49.9 & 31.8 & 3.2 & 5.2 & 23.7 & 69.4 & 150 & 170 & 273.7 \\
\hline & & Coef. Var. & 119 & 73.9 & 81.6 & 76.6 & 73.4 & 136.2 & 225.7 & 156.9 & 151.5 & 96.7 & 94.7 & 104.4 & 29.3 \\
\hline & & Sesgo & 2.8 & 0.8 & 1.3 & 0.5 & 0.8 & 1.7 & 2.3 & 1.8 & 2 & 0.5 & 1 & 1.1 & 0.6 \\
\hline \multirow{5}{*}{$\begin{array}{l}\text { Montejaque } \\
\text { (Central } \\
\text { Eléctrica) }\end{array}$} & \multirow{5}{*}{19} & Media & 83.2 & 58.9 & 64.5 & 56.8 & 44.1 & 17.1 & 0.8 & 1.6 & 16.3 & 47.3 & 80.6 & 113.1 & 584 \\
\hline & & Mediana & 56 & 51 & 53 & 51 & 32 & 8 & 0 & 0 & 3 & 26 & 49 & 84 & 564 \\
\hline & & Desv. Est. & 86.6 & 44 & 46.4 & 48.1 & 48 & 22.1 & 3.4 & 4.6 & 24.7 & 52.8 & 84.4 & 112.4 & 181.4 \\
\hline & & Coef. Var. & 104 & 74.6 & 71.9 & 84.6 & 108.8 & 129.9 & 435.9 & 291.2 & 151.6 & 111.7 & 104.6 & 99.4 & 31 \\
\hline & & Sesgo & 1.7 & 0.5 & 0.6 & 1.3 & 2 & 1.7 & 4.4 & 3.2 & 1.6 & 1.1 & 1.3 & 1.4 & 0.3 \\
\hline \multirow{5}{*}{$\begin{array}{l}\text { Prado del } \\
\text { Rey }\end{array}$} & \multirow{5}{*}{28} & Media & 121 & 88.4 & 65.3 & 71.1 & 59.4 & 19.4 & 2 & 7.3 & 30.6 & 72.7 & 107.5 & 144.1 & 789 \\
\hline & & Mediana & 80.5 & 76.3 & 54 & 60.5 & 49 & 4.8 & 0 & 0 & 20.8 & 64.1 & 102.5 & 113.8 & 744 \\
\hline & & Desv. Est. & 121.6 & 75.7 & 53.1 & 56.7 & 50 & 36.8 & 6.2 & 17.2 & 28.8 & 56.7 & 75.3 & 133.5 & 283.2 \\
\hline & & Coef. Var. & 100.5 & 85.6 & 81.3 & 79.8 & 84.1 & 189.8 & 301.7 & 236.6 & 94.1 & 78 & 70.1 & 92.7 & 35.9 \\
\hline & & Sesgo & 1.7 & 1.1 & 1.8 & 1.3 & 1.2 & 3.4 & 3.5 & 3.8 & 0.6 & 0.4 & 0.7 & 1.6 & 0.9 \\
\hline
\end{tabular}




\begin{tabular}{|c|c|c|c|c|c|c|c|c|c|c|c|c|c|c|c|}
\hline Estación & $\begin{array}{c}\mathrm{N}^{0} \text { años } \\
\text { completos }\end{array}$ & $\begin{array}{l}\text { Estimador } \\
\text { estadístico }\end{array}$ & E & F & M & A & My & $\mathbf{J}$ & Jl & A & S & 0 & $\mathbf{N}$ & D & Año \\
\hline \multirow{5}{*}{$\begin{array}{l}\text { Ronda } \\
\text { (Parchite) }\end{array}$} & \multirow{5}{*}{27} & Media & 83.6 & 103.4 & 65.8 & 61.5 & 29.8 & 8.5 & 1.3 & 1.7 & 26.9 & 72.8 & 83.2 & 104.7 & 643 \\
\hline & & Mediana & 67.8 & 70.7 & 48.5 & 53 & 28 & 1 & 0 & 0 & 16.8 & 71 & 73.2 & 77.7 & 536 \\
\hline & & Desv. Est. & 63.6 & 102.9 & 58.3 & 40.8 & 29.6 & 11.8 & 4.4 & 4.8 & 32.9 & 63.9 & 74.4 & 93.7 & 253.3 \\
\hline & & Coef. Var. & 76 & 99.5 & 88.6 & 66.2 & 99.4 & 139.3 & 346.8 & 290.6 & 122.4 & 87.7 & 89.4 & 89.5 & 39.4 \\
\hline & & Sesgo & 0.9 & 1.8 & 1.4 & 0.6 & 0.9 & 1.2 & 4.4 & 3.8 & 2 & 0.8 & 2.2 & 0.8 & 1 \\
\hline \multirow{5}{*}{$\begin{array}{l}\text { Ubrique } \\
\text { (Instituto) }\end{array}$} & \multirow{5}{*}{13} & Media & 167.4 & 128.5 & 68.7 & 87.5 & 56 & 15.2 & 2.1 & 13.5 & 45.6 & 80.1 & 160.9 & 189.4 & 1.015 \\
\hline & & Mediana & 166.8 & 152.9 & 46.3 & 79.3 & 54.6 & 1.5 & 0 & 0 & 42.2 & 64.4 & 83.8 & 158.6 & 979 \\
\hline & & Desv. Est. & 113.5 & 98.4 & 79 & 53.1 & 40.9 & 26.2 & 5.3 & 36.5 & 39 & 43.2 & 131 & 172.2 & 293.1 \\
\hline & & Coef. Var. & 67.8 & 76.6 & 115 & 60.7 & 73.1 & 172.4 & 252.2 & 270.1 & 85.6 & 54 & 81.4 & 90.9 & 28.9 \\
\hline & & Sesgo & 0.7 & 0.4 & 1.2 & 2.2 & 0.7 & 1.8 & 3.3 & 3.2 & 0.7 & 0 & 0.9 & 0.7 & 0.6 \\
\hline \multirow{5}{*}{$\begin{array}{l}\text { Villaluenga } \\
\text { del Rosario } \\
\text { (ICONA) }\end{array}$} & \multirow{5}{*}{13} & Media & 172 & 165.3 & 151.2 & 129.9 & 61.7 & 32.7 & 2.8 & 4.2 & 72.8 & 188.8 & 251.5 & 279 & 1.512 \\
\hline & & Mediana & 151 & 107 & 105 & 90.6 & 60 & 8 & 0 & 0 & 55.4 & 172 & 171 & 177 & 1.395 \\
\hline & & Desv. Est. & 177.5 & 167.5 & 135.3 & 101.1 & 44.5 & 51 & 6.5 & 7.4 & 44.9 & 104.4 & 227 & 211.4 & 535.7 \\
\hline & & Coef. Var. & 103.2 & 101.3 & 89.5 & 77.8 & 72.1 & 156 & 230.1 & 175.2 & 61.6 & 55.3 & 90.2 & 75.8 & 35.4 \\
\hline & & Sesgo & 1.3 & 1.9 & 0.7 & 1.5 & 1.9 & 1.4 & 2.6 & 2 & 1.1 & 0.6 & 0.9 & 0.6 & 0.6 \\
\hline \multirow{5}{*}{ Villamartín } & \multirow{5}{*}{10} & Media & 62.6 & 65.9 & 71.8 & 62.2 & 31 & 4.9 & 1.1 & 6.6 & 53.3 & 76.1 & 90.6 & 88.4 & 615 \\
\hline & & Mediana & 67.6 & 69 & 53.5 & 55.3 & 16 & 0.6 & 0 & 0 & 56.5 & 66.4 & 84.7 & 45.5 & 663 \\
\hline & & Desv. Est. & 30.8 & 34.3 & 76.5 & 38.4 & 27.6 & 8.2 & 2.4 & 10.7 & 29.8 & 45.3 & 72 & 108.9 & 158 \\
\hline & & Coef. Var. & 49.3 & 52.1 & 106.6 & 61.7 & 88.9 & 166.3 & 211 & 161.5 & 56 & 59.5 & 79.5 & 123.2 & 25.7 \\
\hline & & Sesgo & -0.9 & -0.9 & 2.3 & 2.1 & 1.4 & 2.2 & 1.8 & 1.4 & -0.3 & 0.6 & 0.5 & 2.7 & -0.7 \\
\hline \multirow{5}{*}{$\begin{array}{l}\text { Zahara de la } \\
\text { Sierra (Presa) }\end{array}$} & \multirow{5}{*}{11} & Media & 87.6 & 96.1 & 69.7 & 63.8 & 40.6 & 6.8 & 0.8 & 5 & 38.3 & 75.3 & 70 & 131.8 & 686 \\
\hline & & Mediana & 61 & 85.7 & 63.5 & 47.1 & 30.7 & 2.8 & 0 & 0.2 & 42 & 72.6 & 64.3 & 92.8 & 677 \\
\hline & & Desv. Est. & 58.5 & 85.6 & 50.5 & 57.4 & 29.9 & 10.4 & 2.4 & 7.7 & 28.3 & 44.6 & 58.5 & 139.2 & 230.5 \\
\hline & & Coef. Var. & 66.8 & 89.1 & 72.4 & 90 & 73.6 & 152.5 & 310.6 & 154.4 & 74 & 59.2 & 83.6 & 105.6 & 33.6 \\
\hline & & Sesgo & 0.8 & 2.3 & 0.4 & 2.2 & 0.8 & 2.1 & 3.3 & 1.1 & 0 & 0.9 & 1.7 & 1.8 & 0.7 \\
\hline
\end{tabular}

to, «Cortes de la Frontera, Bañuelos», a $560 \mathrm{~m}$, recibe $1.725 \mathrm{~mm}$; este elevado registro pluviométrico encuentra explicación en la posición favorable que ocupa, abierta a los flujos de procedencia atlántica; mientras que, en sotavento, sorprendentemente, «Villaluenga del Rosario, Icona» $(885 \mathrm{~m})$ y «Grazalema» $(900 \mathrm{~m})$ registran, respectivamente, 1.512 y $1.893 \mathrm{~mm}$.

En la Tabla 4, se encuentra clasificada la precipitación media en las 4 estaciones del año. El período invernal es la época más húmeda del año (precipitación media $>215 \mathrm{~mm}$ ); en todas las estaciones analizadas, salvo en «Villamartín» (35\%), este período representa alrededor del $45 \%$ de la precipitación media anual. El período primaveral constituye entre el $20-25 \%$ de la lluvia media anual; por tanto, si bien este período no representa un porcentaje tan elevado como el invernal, hablamos de una estación relativamente húmeda (precipitaciones medias $>155 \mathrm{~mm}$ ).

El período estival es lo opuesto al invernal, o sea, es la época más seca del año (en general, precipitaciones medias $<40 \mathrm{~mm}$ ); este periodo representa menos del $5 \%$ de las precipitaciones medias anuales. El período otoñal es, tras el invernal, la época más húmeda del año (en general, precipitaciones medias $>180 \mathrm{~mm}$ ), no obstante, hay que destacar que, en ambos periodos, «Villamartín» representa el mismo porcentaje de precipitación media anual (35\%). Dicho esto, todas las estaciones estudiadas constituyen entre el 25-35\% de la lluvia media anual.

Respecto al análisis estadístico (Material suplementario 1) en general, la mediana es inferior a la media. En los meses más húmedos se hallan las mayores diferencias entre ambas medidas de centralidad ( $>30 \mathrm{~mm})$; en cambio, en los más secos se localizan las menores $(<10 \mathrm{~mm})$; respecto a los valores anuales, las diferencias entre estas son, salvo en «Zahara de la Sierra, Presa», superiores a $10 \mathrm{~mm}$, llegando a situarse por encima de los $100 \mathrm{~mm}$ en «Benaocaz», «Cortes de la Frontera, Bañuelos», «Ronda, Parchite» y «Villaluenga del Rosario, Icona».

Existen, sin embargo, en algunas estaciones, meses aislados en que la media es ligeramente inferior a la mediana. Y destaca bastante que, en los valores anuales de «Algodonales, La Muela», «Grazalema»y «Villamartín», 
la mediana es superior a la media. Esto significa que no son las precipitaciones intensas las que sesgan al alza la media sino, todo lo contrario, son las sequías las que la sesgan a la baja (a la inversa de lo esperable en un clima mediterráneo).

Las medidas de dispersión evidencian la enorme variabilidad que tienen, respecto a la media, los datos pluviométricos. Esta variabilidad se manifiesta en todas las medidas de dispersión seleccionadas: la desviación estándar presenta valores elevados, que serán más elevados a medida que nos acerquemos a los meses invernales; los meses con mayor coeficiente de variación son los estivales (valores muy dispersos: $>100 \%$ ) que desvirtúan la media en dicha época; toda esta variabilidad de los datos pluviométricos se refuerza con un sesgo positivo muy alto (entre 1 y 5). Por otro lado, en los datos anuales la desviación estándar registra, en todas las estaciones, valores muy altos $(>150)$, que en «Cortes de la Frontera, Bañuelos» y «Grazalema» superan los 500; en cambio, la variabilidad anual, aunque sigue siendo considerable, es menor que la mensual; el coeficiente de variación oscila entre $25-40 \%$ (datos heterogéneos) y el sesgo es, salvo en «Villamartín» donde es negativo (-0.7), positivo y moderado, oscilando entre 0.2 y 1 .

\subsubsection{Número medio de días de precipitación e intensidad}

En la Figura 5 se representan gráficamente dos de los aspectos más relevantes del análisis pluviométrico: número medio mensual de días de precipitación e intensidad

Tabla 4: Pluviometría media estacional y porcentaje (\%) de precipitación respecto al total anual de las 13 estaciones climáticas utilizadas en el análisis pluviométrico. Fuente: elaboración propia.

Table 4: Average rainfall and percentage (\%) of precipitation with respect to the annual total of the 13 climatic stations used in the pluviometric analysis. Source: own elaboration.

\begin{tabular}{|c|c|c|c|c|c|c|}
\hline Estación & Variables & Invierno & Primavera & Verano & Otoño & Anual \\
\hline \multirow{2}{*}{ Algodonales (La Muela) } & $\mathrm{mm}$ & 316.3 & 176.7 & 25.6 & 183.4 & 702 \\
\hline & $\%$ & 45.1 & 25.2 & 3.6 & 26.1 & 100 \\
\hline \multirow{2}{*}{ Benaocaz } & $\mathrm{mm}$ & 642.6 & 375.6 & 63.9 & 333.6 & 1.416 \\
\hline & $\%$ & 45.4 & 26.5 & 4.5 & 23.6 & 100 \\
\hline \multirow{2}{*}{ Benaoján (Cueva de la Pileta) } & $\mathrm{mm}$ & 623.4 & 298.4 & 28 & 372.5 & 1.322 \\
\hline & $\%$ & 47.2 & 22.6 & 2.1 & 28.2 & 100 \\
\hline \multirow{2}{*}{ Cortes de la Frontera (Bañuelos) } & $\mathrm{mm}$ & 776.6 & 383.2 & 40.6 & 524.6 & 1.725 \\
\hline & $\%$ & 45 & 22,2 & 2,4 & 30,4 & 100 \\
\hline \multirow{2}{*}{ Grazalema } & $\mathrm{mm}$ & 867.8 & 514.9 & 24.6 & 485.7 & 1.893 \\
\hline & $\%$ & 45.8 & 27.2 & 1.3 & 25.7 & 100 \\
\hline \multirow{2}{*}{ Jimera de Líbar (Central eléctrica) } & $\mathrm{mm}$ & 421 & 238.9 & 28 & 245,8 & 934 \\
\hline & $\%$ & 45.1 & 25.6 & 3 & 26.3 & 100 \\
\hline \multirow{2}{*}{ Montejaque (Central eléctrica) } & $\mathrm{mm}$ & 255.2 & 165.4 & 19.5 & 144.2 & 584 \\
\hline & $\%$ & 43.7 & 28.3 & 3.3 & 24.7 & 100 \\
\hline \multirow{2}{*}{ Prado del Rey } & $\mathrm{mm}$ & 353,5 & 195,8 & 28,7 & 210,8 & 789 \\
\hline & $\%$ & 44.8 & 24.8 & 3.6 & 26.7 & 100 \\
\hline \multirow{2}{*}{ Ronda (Parchite) } & $\mathrm{mm}$ & 291.7 & 157.1 & 11.5 & 182.9 & 643 \\
\hline & $\%$ & 45.4 & 24.4 & 1.8 & 28.4 & 100 \\
\hline \multirow{2}{*}{ Ubrique (Instituto) } & $\mathrm{mm}$ & 485.3 & 212.2 & 30.8 & 286.6 & 1.015 \\
\hline & $\%$ & 47.8 & 20.9 & 3 & 28.2 & 100 \\
\hline \multirow{2}{*}{ Villaluenga del Rosario (ICONA) } & $\mathrm{mm}$ & 616.3 & 342.8 & 39.7 & 513.1 & 1.512 \\
\hline & $\%$ & 40.8 & 22.7 & 2.6 & 33.9 & 100 \\
\hline \multirow{2}{*}{ Villamartín } & $\mathrm{mm}$ & 216.9 & 165 & 12.6 & 220 & 615 \\
\hline & $\%$ & 35.3 & 26.8 & 2 & 35.8 & 100 \\
\hline \multirow{2}{*}{ Zahara de la Sierra (Presa) } & $\mathrm{mm}$ & 315.5 & 174.1 & 12.6 & 183.6 & 686 \\
\hline & $\%$ & 46 & 25.4 & 1.8 & 26.8 & 100 \\
\hline
\end{tabular}




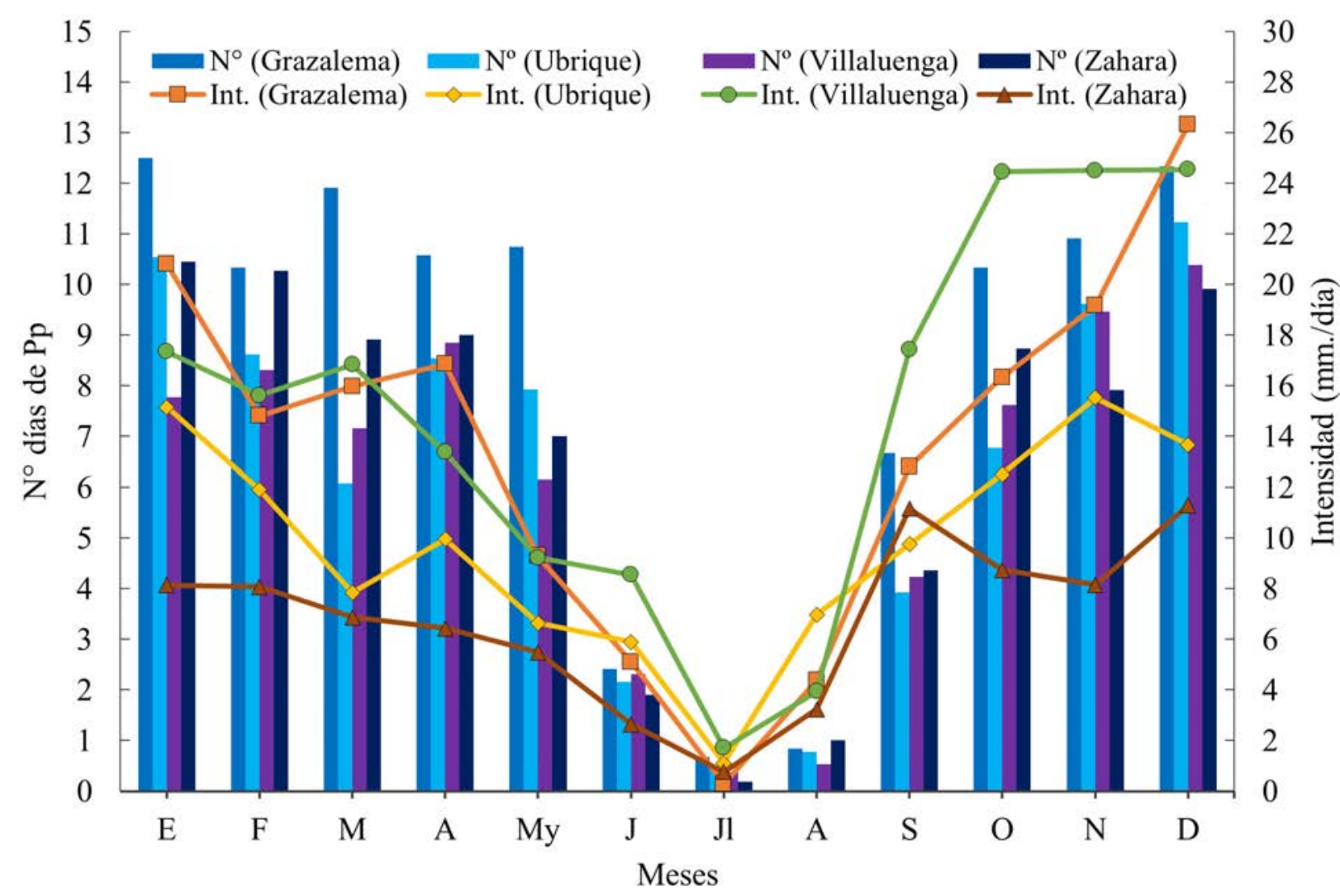

Figura 5: Número medio mensual de días de precipitación $\left(\mathrm{N}^{\circ}\right)$ e intensidad media diaria mensual (Int.) de precipitación (Grazalema, Ubrique, Villaluenga y Zahara). Fuente: elaboración propia.

Figure 5: Average monthly number of precipitation days $\left(N^{\circ}\right)$ and monthly average daily intensity (Int.) of precipitation (Grazalema, Ubrique, Villaluenga and Zahara). Source: own elaboration.

media diaria mensual de las 4 estaciones ubicadas en el interior del Parque («Grazalema», «Ubrique, Instituto», «Villaluenga del Rosario, Icona» y «Zahara de la Sierra, Presa»).

En La Figura 5 se aprecia que el número medio mensual de días de precipitación y la intensidad media diaria mensual presentan un marcado contraste estacional, con un máximo invernal (diciembre o enero) y un claro mínimo estival (julio). El número medio mensual de días de precipitación es, entre octubre y mayo, $>6$ en todas las estaciones; de ellas, "Grazalema» es, con diferencia, la que presenta en este período un mayor número medio de días de precipitación $(>10)$; siendo la estación con el mayor volumen pluviométrico medio anual. En las 3 estaciones restantes el número medio de días de precipitación es similar, sin una diferencia mayor entre ellas de 3 días en cada mes. Dicho esto, es llamativo que «Zahara de la Sierra, Presa» y «Ubrique, Instituto», las estaciones con los volúmenes pluviométricos medios anuales más bajos, tengan, en la mayoría de estos meses, más días de lluvia que «Villaluenga del Rosario, Icona», ya que esta última las supera en 700 y $500 \mathrm{~mm}$ medios anuales respectivamente.

Durante los meses estivales y el primero del otoño el número medio mensual de días de precipitación es $<5$, propiciado por los casi inexistentes registros pluviométricos de dicho período, con valores homogéneos en todas las estaciones, excepto en septiembre, donde «Grazalema» registra cerca de 7 días ( 2 más que las restantes estaciones).

En general, el volumen pluviométrico medio mensual es, en este caso, la variable con mayor repercusión en la intensidad media diaria mensual; esta variable será menor en las estaciones consideradas cuanto menos volumen pluviométrico medio anual registren; esto es así, especialmente, en «Zahara de la Sierra, Presa» y «Ubrique, Instituto», siendo la primera la de menor intensidad media diaria mensual. Estas se caracterizan por ser precipitaciones débiles, pues, excepto en septiembre y noviembre, se sitúan en $<10 \mathrm{~mm} /$ día; «Ubrique, Instituto», aunque es la segunda estación con menor intensidad media diaria mensual (la mayoría de meses), presenta lluvias entre débiles y moderadas (4-16 mm/día). Los valores de las 2 estaciones con mayor intensidad media diaria mensual son «Grazalema» y «Villaluenga del Rosario, Icona». Estas estaciones presentan lluvias moderadas (10-26 mm/día), salvo entre mayo y agosto donde son débiles $(<10 \mathrm{~mm} /$ día). La intensidad media anual de «Villaluenga del Rosario, Icona» es, en la mayoría de meses, ligeramente superior a la de «Grazalema», pese a tener un volumen pluvio- 
métrico anual $300 \mathrm{~mm}$ inferior a esta. No obstante, la mayor intensidad media diaria mensual se registra en «Grazalema», en diciembre (26 mm/día).

Por otro lado, se han analizado todos los eventos pluviométricos que se han registrado en la estación de «Grazalema» en el período 1992-2015. Estos han sido un total de 1.955 eventos que han arrojado los siguientes valores de intensidad (Figura 6): De los 1.955 eventos, 37 han registrado intensidades superiores a $100 \mathrm{~mm}$ en 24 horas, lo que supone un $2.5 \%$ de los días lluviosos a lo largo de estos 22 años. Si se considera que el número medio de días lluviosos al año es de 88.3 en Grazalema, el 2.5\% supone un promedio de más de dos eventos superiores a $100 \mathrm{~mm}$ al año.

Empleando la distribución de Poisson para estos cálculos se obtiene una probabilidad del $41 \%$ de registrar al menos un caso $>100 \mathrm{~mm}$ cada año. Llama la atención el hecho de que la probabilidad de que se registren al menos 2 casos alcanza casi el $35 \%$ y el $19.5 \%$ para al menos 3 casos. Si atendemos a la realidad de los eventos, centrándonos solo en aquellos que superaron los $150 \mathrm{~mm}$ (12 casos), estos se produjeron en 9 de los 22 años registrados, lo que implica que algunos años se registraron varios casos de más de $150 \mathrm{~mm}$.

Se han clasificado estos eventos en función de los aspectos de la dinámica atmosférica, alcanzando los siguientes resultados (Material suplementario 2): En primer lugar, destaca que la mayor parte de los eventos responden a masas de aire atlánticas inestabilizadas al entrar en la Península. Ninguno de los casos responde a eventos de inestabilidad en altura típicos del Mediterráneo (DANAs), aunque en varios casos la intervención de flujos fríos en altura, la oclusión de frentes o la fuerte actividad de borrascas dinámicas revelan intervenciones de las masas de aire en niveles medios. Igualmente, en ningún caso se produce flujo mediterráneo (levante).
El gradiente térmico inicial es variable, aunque de escasa fiabilidad, dados los enormes cambios que pueden sufrir en un mismo día.

Los 11 eventos señalados en el Material suplementario 2 incluyen uno (21-26/12/2009) con dos episodios de lluvias de más de $150 \mathrm{~mm}$ consecutivos (24 y 25/12/2009). Así, se llegan a alcanzar en un único evento de más de 24 horas de duración volúmenes que superan los $400 \mathrm{~mm}$ (marzo de 2013) y los $740 \mathrm{~mm}$ en unos pocos días, respectivamente.

En «Grazalema» uno de los eventos analizados (7$8 / 2 / 2007$ ) se aproxima a $220 \mathrm{~mm}$, lo que supone un $23.7 \%$ de las lluvias de todo el año. Sin embargo, lo más habitual es que se sitúen por debajo del $15 \%$ del total de precipitaciones anuales.

\subsection{Gradientes y cartografía}

La Figura 7 muestra la representación altitudinal del gradiente pluviométrico en solana y umbría, la cual se limitó a la precipitación media anual. Ante los elevados coeficientes de correlación obtenidos en todos los gradientes calculados, se demuestra que son aplicables de modo generalizado.

Se aprecia, cómo en solana la precipitación media anual aumenta más rápidamente con la altitud que en umbría $(1.72 \mathrm{~mm} / \mathrm{m}$ y $1.30 \mathrm{~mm} / \mathrm{m}$, respectivamente). En la solana, el ascenso es de $2.700 \mathrm{~mm}$ en $1.600 \mathrm{~m}$; en cambio, en la umbría es de $2.000 \mathrm{~mm}$ en el mismo desnivel. Aunque, a $0 \mathrm{~m}$, la umbría es más húmeda que la solana (150 y $350 \mathrm{~mm}$, respectivamente), al alcanzar los $400 \mathrm{~m}$ las dos registran el mismo valor pluviométrico $(850 \mathrm{~mm}) \mathrm{y}$, rebasada dicha altitud, la solana es más húmeda que la umbría.

En la Figura 8, utilizando el método IDW, se puede observar el trazado que las isoyetas muestran a lo largo

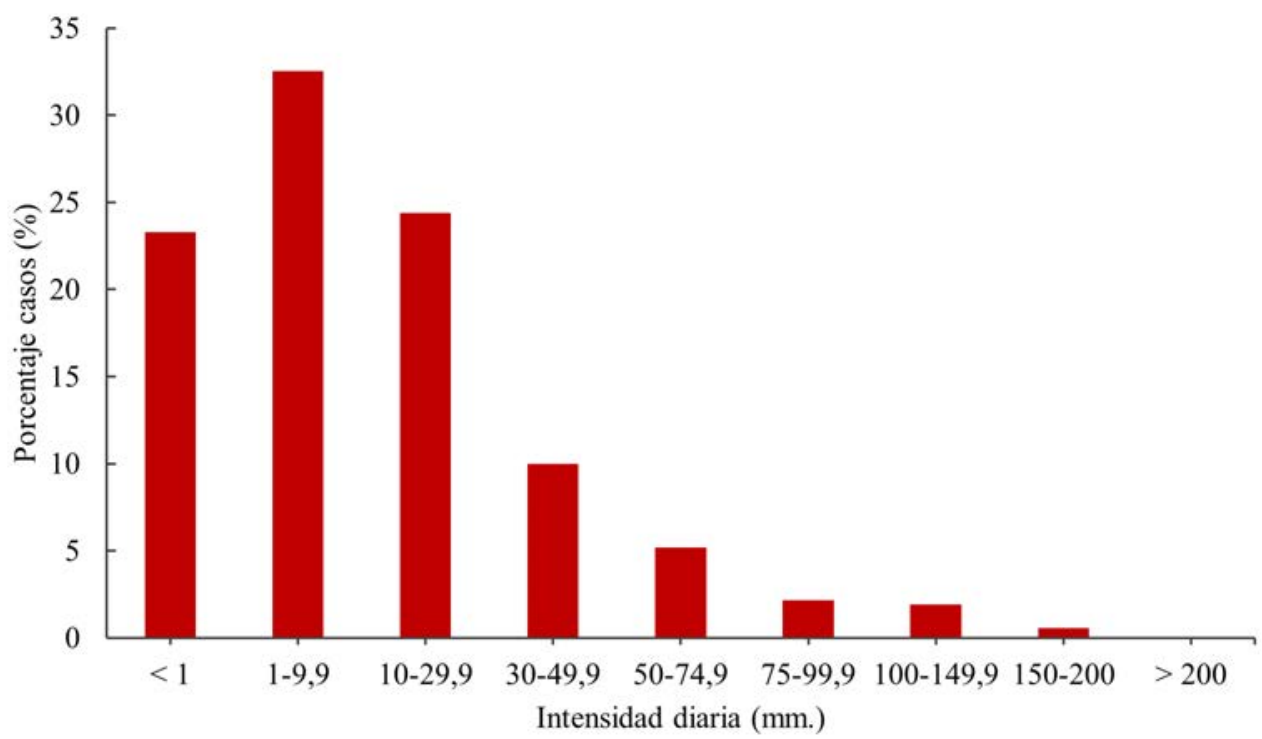

Figura 6: Distribución de las lluvias en Grazalema según su volumen en 24 h. Fuente: elaboración propia. Figure 6: Distribution of rains in Grazalema according to their volume in $24 \mathrm{~h}$. Source: own elaboration. 
Material suplementario 2: Eventos pluviométricos torrenciales estación Grazalema (E198), clasificados en función de los aspectos de la dinámica atmosférica. Fuente: elaboración propia. Leyenda: F= Frío; B= Borrasca; $\mathrm{C}=\mathrm{Cálido}$; Oc= Ocluido.

\begin{tabular}{|c|c|c|c|c|c|c|c|c|}
\hline Fecha(s) Evento & Situación atmosférica & $\begin{array}{c}\text { Dir. } \\
\text { Viento }\end{array}$ & $\begin{array}{c}\text { T. }\left({ }^{\circ} \mathbf{C}\right) \\
\text { Superficie }\end{array}$ & $\begin{array}{c}\text { T. }\left({ }^{\circ} \mathbf{C}\right) \\
\mathbf{8 5 0 ~ H P a . ~}\end{array}$ & $\begin{array}{c}\text { Vol. (mm.) total } \\
\text { precipitado }\end{array}$ & $\begin{array}{c}\text { Máxima } \\
\text { precipitación en } \\
\mathbf{2 4} \text { horas (mm) }\end{array}$ & $\begin{array}{c}\text { Máxima } \\
\text { precipitación } \\
\text { horaria (mm) }\end{array}$ & $\begin{array}{c}\text { Total } \\
\text { anual } \\
(\mathbf{m m})\end{array}$ \\
\hline $05-06 / 01 / 1996$ & Frente Frío & W-SW & 16 & 6 & 270.9 & 161.9 & 28.3 & 3.212 \\
\hline $11-12 / 11 / 1996$ & Frente F+ Flujo frío + B. activa & SW & 16 & 8 & 257.8 & 186.3 & 24.5 & 3.212 \\
\hline $20 / 10 / 1999$ & Frente F+ B. activa & W-SW & 16 & 10 & 201.4 & 188.8 & 25.3 & 1.397 \\
\hline $26-27 / 01 / 2001$ & Frente C+F & W-NW & 14 & 6 & 218 & 174.2 & 11.9 & 2.020 \\
\hline $07-08 / 02 / 2007$ & Frente C+F & W-SW & 15 & 5 & 217.4 & 190 & 18.4 & 916 \\
\hline $31 / 10-1 / 11 / 2008$ & Frente C+F+ B. activa & SW & 14 & 6 & 195.7 & 174.7 & 15.6 & 1.977 \\
\hline $21-26 / 12 / 2009$ & Frente C+F+ B. activas & SW & 17 & 7 & 749.5 & 161.8 & 27.7 & 2.812 \\
\hline $14-15 / 12 / 2012$ & Frente C+F & NW & 13 & 3 & 217.8 & 196.1 & 15.7 & 1.525 \\
\hline $05-08 / 03 / 2013$ & Frente Oc+F & SW & 13 & 8 & 404.2 & 186.7 & 24.6 & 2.468 \\
\hline $25 / 12 / 2013$ & Frente Oc+F & W-SW & 13 & 5 & 237.2 & 237.2 & 35.1 & 2.468 \\
\hline $08-09 / 02 / 2014$ & Frente Oc+F & W-SW & 11 & 3 & 302.6 & 191.9 & 27 & 2.367 \\
\hline
\end{tabular}

de la sierra. Los resultados muestran un mapa detallado y se corresponde, a nuestro parecer, con la realidad del espacio representado, considerando los cambios de gradientes y adaptándose al relieve. En las cumbres de la Sierra de Líbar, Pinar y Endrinal, en zonas $>1.400 \mathrm{~m}$ s.n.m., localizamos los valores de precipitación media anual más elevados del área de estudio con valores superiores a los $2.400 \mathrm{~mm}$. Bordeando los núcleos orográficos de la Sierra de Grazalema, en las zonas más bajas
( $<500$ m s.n.m.), se registran los mínimos de precipitación media anual $(<600 \mathrm{~mm})$.

\subsection{Efecto Venturi}

Partiendo de la ecuación de Bernoulli, por ejemplo, se constata que una masa de aire que entra desde Tavizna, a $3 \mathrm{~m} / \mathrm{s}$, asciende al Puerto del Boyar y luego desciende

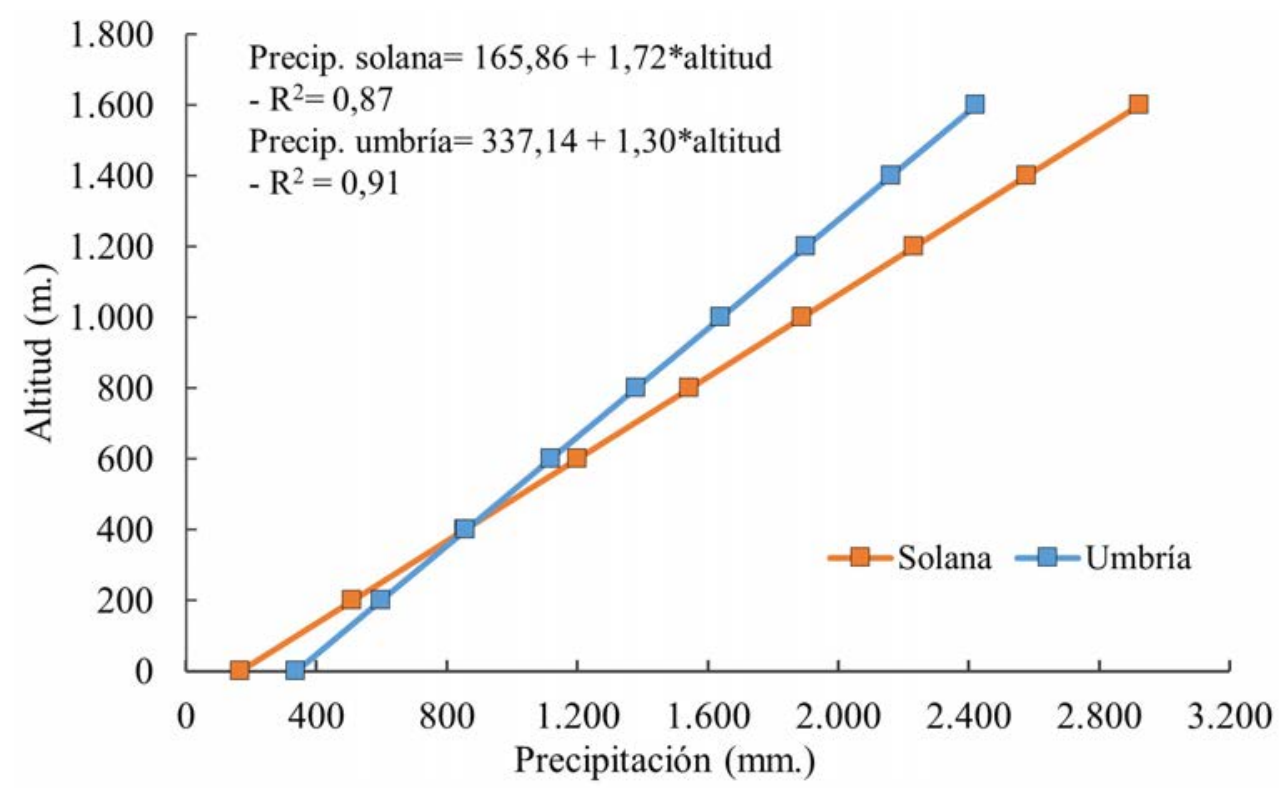

Figura 7: Representación altitudinal del gradiente pluviométrico medio anual en las laderas de solana y umbría. Leyenda: Gradiente pluviométrico medio anual de solana $=1.72 \mathrm{~mm} / \mathrm{m}$; gradiente pluviométrico medio anual de umbría $=1.30 \mathrm{~mm} / \mathrm{m}$. Fuente: elaboración propia.

Figure 7: Altitudinal representation of the mean annual rainfall gradient on the slopes of suntrap and umbria. Legend: Average annual rainfall gradient in suntrap $=1.72 \mathrm{~mm} / \mathrm{m}$; Average annual rainfall gradient in umbría $=1.30 \mathrm{~mm} / \mathrm{m}$. Source: own elaboration. 


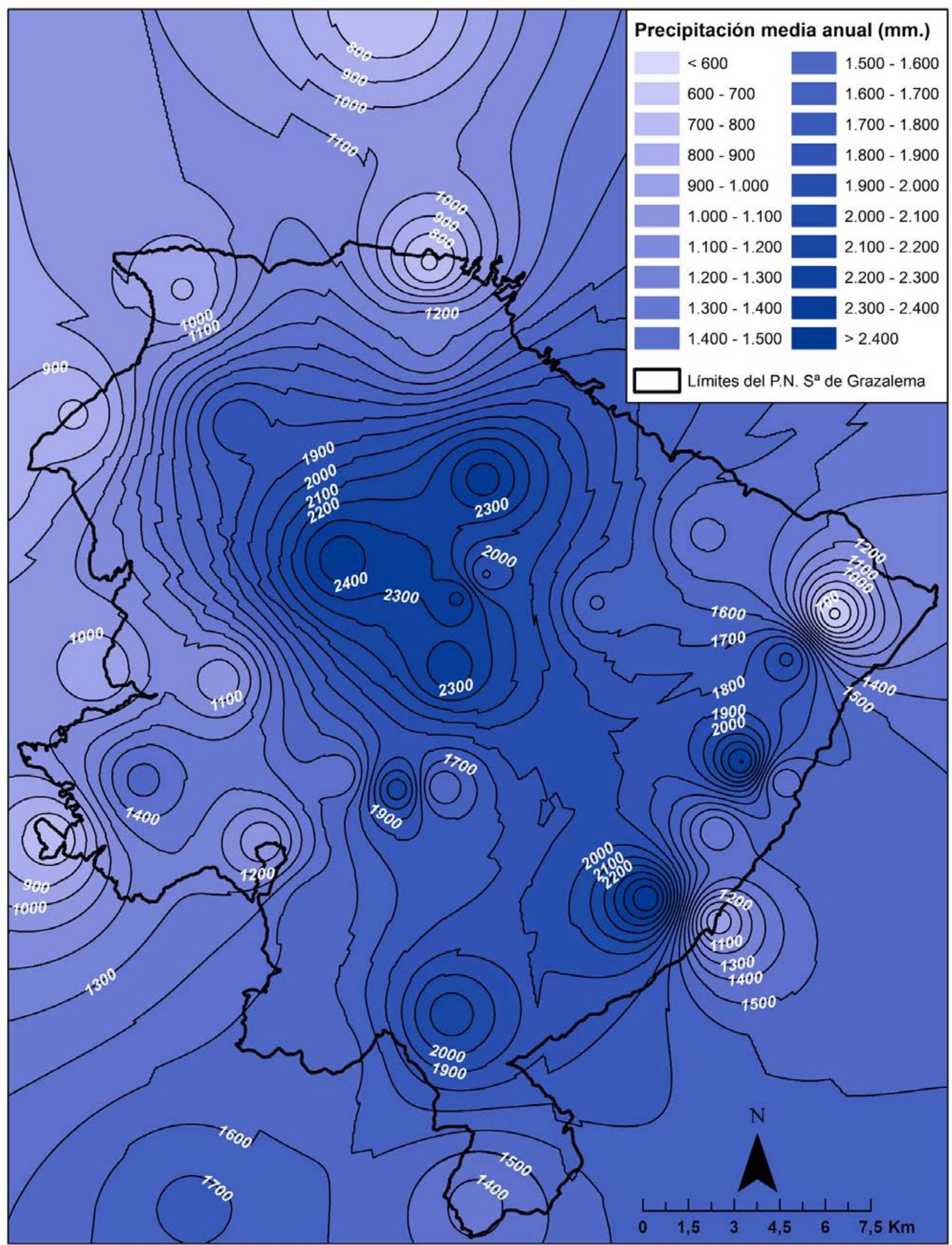

Figura 8: Mapa de precipitación. Método interpolación IDW. Fuente: elaboración propia a partir de datos del IECA. Figure 8: Precipitation map. IDW interpolation method Source: based on the IECA. 
hasta Grazalema helipuerto, multiplica su velocidad en gran medida, alcanzando los $13.52 \mathrm{~m} / \mathrm{s}$ (Tabla 3 ).

A partir de ello, el efecto Venturi implica que, dado que la velocidad aumenta, la presión debe disminuir. Empleando la misma fórmula, una presión inicial de 888 HPa (presión media a $1.100 \mathrm{~m}$. de altura, es decir, en el Puerto del Boyar) descendería en Grazalema helipuerto hasta $773 \mathrm{HPa}$ (altura que vendría a corresponderse a la presión teórica próxima a $2.200 \mathrm{~m}$ ).

Al calcular la cantidad de agua precipitable comparando las diferencias de presión entre uno y otro punto, se produce un incremento horizontal de $1.181 / \mathrm{m}^{2}$, al que habría que sumar la altura de la masa nubosa, que podrá incrementarse como consecuencia de la menor presión y, con ello, mayor posibilidad de ascendencias.

Así, siguiendo el mismo ejemplo anterior, una masa de aire de $4.000 \mathrm{~m}$. de altura con $888 \mathrm{HPa}$ en la base, en el Puerto del Boyar alcanzaría un valor de agua precipitable de $1.69 \mathrm{l} / \mathrm{m}^{2}$. O lo que es lo mismo, un incremento del $43 \%$ de la posibilidad de precipitación en Grazalema helipuerto respecto al Puerto del Boyar (Tabla 3), valor próximo a la realidad de los registros pluviométricos (hay que destacar que, por ejemplo, en Benaocaz caen 1.416 1/año, frente a los 1.893 1/año de Grazalema, es decir, un 33.7\% más).

Sin embargo, al abrirse el valle a partir de Grazalema, se va produciendo una reducción drástica respecto al helipuerto; reducción que, no obstante, sigue implicando un incremento de precipitaciones respecto al Puerto del Boyar. En el caso de Grazalema estación supone un incremento del 5\% respecto al Boyar, que se reduce al 2.3\% en Grazalema Ayuntamiento (Tabla 3). Si continuásemos un poco más abajo, las precipitaciones serían claramente inferiores a las del Boyar.

En cambio, cuando se atiende a los mismos efectos en los otros corredores analizados (Manga de Villaluenga y Pasillo de Líbar), los resultados no son similares. Así, entre Boca de Villaluenga (entrada al corredor desde Benaocaz) y Villaluenga estación, el resultado muestra un $4.8 \%$ menos de precipitación en el segundo punto, valores negativos que también se dan entre Puerto de Villaluenga y Villaluenga estación, y entre Boca de Villaluenga y Puerto de Villaluenga (Tabla 3). Esto implica que debería llover más en la Boca de Villaluenga que en el núcleo de Villaluenga, donde se sitúa la estación meteorológica.

En el caso de Líbar, se ha comparado el Cortijo de Líbar con otros puntos del valle, más al NE. El estrechamiento de la Fuente de Cufría apenas muestra diferencias con el Cortijo ( $0.5 \%$ más de precipitación) y la entrada del valle desde Montejaque poco más ( $1.5 \%$ de incremento) (Figura 3).

Tras este análisis, puede inferirse que el caso de Grazalema es excepcional, ya que implica un estrechamiento del valle a sotavento, a menor altura, pero con mucha menos superficie de aforo de aire, lo que implica un incremento de la velocidad y una disminución de la presión atmosférica. El efecto Venturi se pone de manifiesto en los resultados expuestos en la Tabla 3 .

\section{Discusión}

Es un hecho constatado que los factores geográficos originan alteraciones en las variables climáticas; los más importantes, a nivel general, son: la latitud, la longitud y la topografía. La topografía, a mesoescala, ejerce una influencia muy importante sobre el clima (Capel, 2000), pues se puede afirmar que las precipitaciones aumentan con la altitud (Cuadrat \& Pita, 2006), mientras que las temperaturas disminuyen con esta (Aparicio \& Silvestre, 1996); además de la altitud, otros factores topográficos que modifican las características climáticas locales son: la exposición y la orientación de las laderas (solana-umbría, barlovento-sotavento, etc.) (Aguiló, 2004; Quereda, 2005).

La exposición de las laderas genera importantes diferencias pluviométricas entre barlovento y sotavento; barlovento se orienta a favor de los vientos dominantes y sotavento a resguardo de estos. Considerando la posición de ambas laderas, es lógico ratificar lo que señala la «lluvia orográfica»: que los vientos dominantes, en este caso, los de componente oeste, se ven obligados a ascender por barlovento, condensando y descargando en forma de lluvia en dicha ladera, mientras que sotavento no recibe precipitaciones (Vázquez, 2010); pero, en ocasiones, en latitudes medias y altas, se producen depresiones de sotavento (de origen no frontal).

Estas se originan, «en situaciones en que una corriente procedente del oeste es forzada a ascender, más o menos transversalmente, sobre una barrera de montañas orientadas en dirección norte-sur» (Quereda, 2005); este movimiento vertical genera una divergencia con curvatura anticiclónica en la cumbre de la montaña, y convergencia con curvatura ciclónica a sotavento (Casas \& Alarcón, 1999; Quereda, 2005).

Grazalema está al este del conjunto montañoso (sierras del Pinar, Endrinal, Caíllo y de Ubrique) y, como Villaluenga del Rosario, en una posición intramontana respecto a los vientos del sur y del norte; posición por la cual decir que está al norte o al sur del conjunto montañoso induce a error en ambos casos; aun así, la posición de Grazalema y, algo menos, de Villaluenga del Rosario, es de sotavento para vientos del oeste y del sur, si se observan las altitudes de los conjuntos que tiene Grazalema hacia estas direcciones. Dicho esto, el análisis pluviométrico demuestra que, tanto Grazalema como Villaluenga del Rosario, registran precipitaciones medias anuales superiores a las de los municipios del Parque situados a barlovento (Benaocaz y Ubrique).

En la misma línea, López et al. (1989) y Martín (1991) relacionan el hecho de que el pueblo de Grazalema, al este del Puerto del Boyar y a sotavento, registre precipitaciones más elevadas que los municipios ubicados en barlovento, con un efecto Venturi provocado por «la canalización de los flujos atlánticos por el mencionado puerto, embudo de las sierras del Pinar, al norte, y del Endrinal, al sur» (Martín, 1991).

Diversos trabajos han corroborado que una masa de aire, al pasar por un estrechamiento, aumenta su velocidad y disminuye su presión por efecto Venturi (Dorman et 
al., 1995; Zúñiga \& Crespo del Arco, 2010). Sin embargo, tanto la afirmación realizada por López et al. (1989) como la suposición de Martín (1991) podrían ser inexactas; puesto que, Martín (1991) sólo intuye sin llevar a cabo ningún cálculo, y el análisis que realizan López et al. (1989) es de una situación meteorológica (aunque esta se prolongue durante varios días) y, por tanto, no podría alcanzar una validez climática suficiente.

Dicho esto, profundizando, de un modo más científico con cálculos y mediciones en los valles, y como se ha comprobado en el epígrafe 3.3., se puede afirmar que la diferencia de ancho del valle del Corredor del Boyar hasta Grazalema provoca un efecto Venturi por estrechamiento y, con ello, una reducción de la presión atmosférica que facilita ascendencias más fuertes a sotavento que a barlovento, como se explica de modo pormenorizado a continuación (Figura 9): la entrada al Corredor del Boyar desde Tavizna tiene 1,6 km de ancho; luego se abre en el Salto del Cabrero (casi $2 \mathrm{~km}$ de ancho); al llegar al puerto del Boyar, el valle no llega a $1 \mathrm{~km}$ de ancho (comienza el efecto Venturi); cerca del cruce de Zahara tiene $500 \mathrm{~m}$ (pero más abajo en cota; el efecto Venturi se acentúa); el ancho del valle es el mismo en el punto medio entre el cruce de Zahara y el campo de fútbol: $500 \mathrm{~m}$; sobre el propio Helipuerto, el valle se estrecha aún más, y alcanza 400 m (acentúa el efecto Venturi disminuyendo aún más la presión) y ya en el núcleo municipal de Grazalema se abre hasta 700 m (más estrecho que en el Salto del Cabrero), amplitud que se mantiene justo hasta el mirador de
Grazalema, a partir de donde se abre de modo manifiesto el valle.

Con esto, la hipótesis inicial en Grazalema queda validada: los elevados registros pluviométricos medios anuales de «Grazalema», más cuantiosos que en estaciones situadas a barlovento, se deben a un efecto de «depresión de sotavento» (con refuerzo de efecto Venturi), producido por el Corredor del Boyar.

En el caso de la Manga de Villaluenga se cumple la hipótesis, aunque no se produce un efecto Venturi tan marcado como en Grazalema. En el Pasillo de Líbar las diferencias entre los puntos estimados son mínimas, lo que implica una hipótesis nula en este caso.

Respecto al análisis de los eventos pluviométricos que se han registrado en la estación de «Grazalema», en el período 1992-2015, podemos señalar que en este tipo de análisis la rigurosidad de los intervalos siempre limita el estudio, ya que, es necesario señalar que en Grazalema un evento de más de $100 \mathrm{~mm}$ en un día puede venir sucedido de otro semejante varios días después y que un evento de más de $20 \mathrm{~mm}$ en una hora puede venir sucedido de varios eventos semejantes en las horas sucesivas del mismo día o del mismo evento lluvioso. Ambos hechos implican una importante acumulación de agua en muy cortos periodos de tiempo, pero no son catalogados como torrenciales en ningún caso. Pero habría que considerar si un evento $>200 \mathrm{~mm}$ en 24 horas, pero $<60 \mathrm{~mm} / \mathrm{h}$ que requiere AEMET para ser considerado torrencial, debe o no ser considerado como tal,

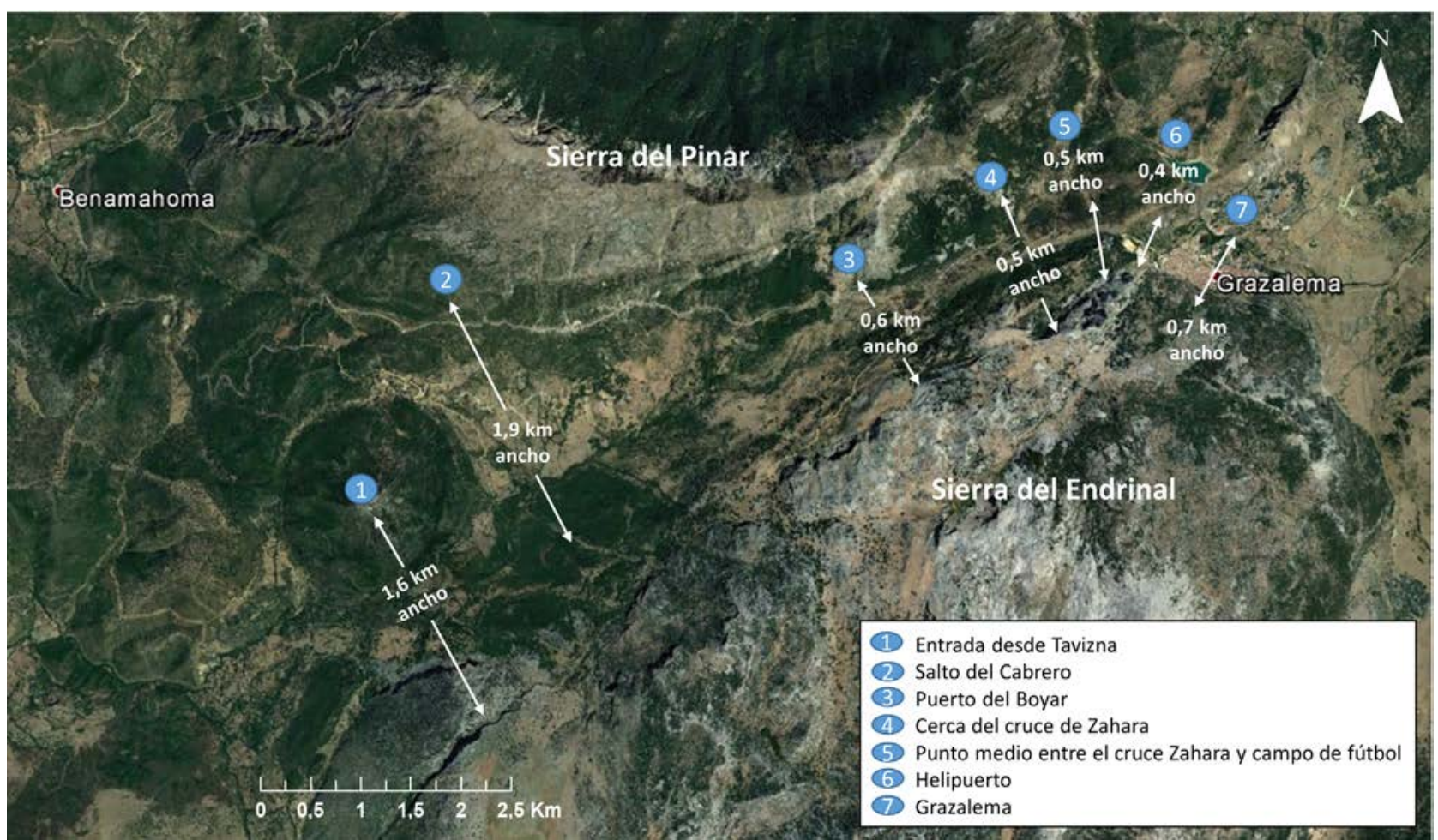

Figura 9: Mediciones en el Corredor del Boyar. Fuente: elaboración propia a partir de imagen satélite de Google Earth (2017). Figure 9: Measurements in the Boyar Corridor. Source: based on the satellite image of Google Earth (2017). 
dado que la intensidad promedio mantenida $>10 \mathrm{~mm} /$ hora a lo largo de todo el evento de 24 horas es digna de ser considerada, tal vez, por encima de la categoría de «muy fuerte».

\section{Conclusiones}

En el presente trabajo como principales conclusiones hemos encontrado que:

- Existe un problema en la definición de barloventosotavento en la Sierra de Grazalema: aunque, como parece lógico, en general, se aprecia mayor pluviometría media anual en la vertiente de barlovento que en la de sotavento, existen unas «islas» pluviométricas marcadas por «Grazalema»y «Villaluenga del Rosario, Icona» que rompen dicha lógica ya que estas, a sotavento, registran, respectivamente, el primer y el tercer valor pluviométrico anual más elevado.

- Estadísticamente, la precipitación anual de «Algodonales, La Muela», «Grazalema» y «Villamartín», posee una mediana superior a la media; esto significa que, en dichas estaciones, las precipitaciones intensas no sesgan al alza la media sino al contrario, son las sequías las que la sesgan a la baja: al contrario de lo esperable en un clima mediterráneo.

- Se demuestra que con la creación de puntos climatológicos extrapolados georreferenciados y con valores pluviométricos y el uso del interpolador IDW, se logra obtener una cartografía climática de mayor precisión, pues este mapa se ajusta, a nuestro parecer, mejor al relieve.

- Los elevados registros pluviométricos anuales que se registran en la estación de «Grazalema» no responden a una posición de barlovento como suele señalarse, sino a un efecto de depresión de sotavento (con refuerzo de efecto Venturi), producido por el Corredor del Boyar. Tras este análisis, puede inferirse que el caso de Grazalema es excepcional, ya que implica un estrechamiento del valle a sotavento, a menor altura, pero con mucha menos superficie de aforo de aire, lo que implica un incremento de la velocidad y una disminución de la presión atmosférica. El efecto Venturi incrementa la posibilidad de precipitación, a la salida del Puerto del Boyar (Grazalema helipuerto) en un $43 \%$ respecto al inicio del Corredor.

- Es necesario proceder a la medición exacta de las diferencias pluviométricas entre el Puerto del Boyar y Grazalema con datos reales (instalación de aparatos de medición), para corroborar o refutar los resultados de las mediciones realizadas en este estudio; este es el objetivo de futuras investigaciones en esta línea.

\section{Referencias}

Aguiló, M. (Coord.), 2004. Guía para la elaboración de estudios del medio fisico. Contenido y metodología ( $5^{\mathrm{a}}$ Reimpresión).
Centro de Publicaciones. Secretaría General Técnica. Ministerio de Medio Ambiente, Madrid.

Albentosa, L.M., 1989. El clima y las aguas. Editorial Síntesis, D.L., Madrid.

Albentosa, L.M., 1990. Climatología y Medio Ambiente. Servicio de Publicaciones de la Universitat de Barcelona., Barcelona.

Aparicio, A. \& Silvestre, S., 1996. Guía de la flora y la fauna del Parque Natural Sierra de Grazalema. Consejería de Medio Ambiente, Junta de Andalucía, Jerez.

Atkinson, P.M. \& Lloyd, C.D., 1998. Mapping precipitation in Switzerland with ordinary and indicator kriging. Journal of Geographic Information and Decision Analysis, 2: 65-76.

Bannister, D. \& King, J., 2015. Föhn winds on South Georgia and their impact on regional climate. Weather, 70: 324-329. Doi: $10.1002 /$ wea.2548.

Bustamante, J., 2003. Cartografía predictiva de variables climáticas: comparación de distintos modelos de interpolación de la temperatura en la España Peninsular. Graellsia, 59 (2-3): 359-376. Doi: http://dx.doi.org/10.3989/graellsia.2003.v59. i2-3.252.

Capel, J.J., 2000. El clima de la península ibérica. Ariel, Barcelona.

Casas, M.C. \& Alarcón, M., 1999. Meteorología y clima. Edicions UPC, Barcelona.

Cerdà, A., 1998. Relationships between climate and soil hydrological and erosional characteristics along climatic gradients in Mediterranean limestone areas. Geomorphology, 25: 123-134. Doi: 10.1016/S0169-555X(98)00033-6.

Cuadrat, J.M. \& Pita, M.F., 2006. Climatología (4a ed.). Editorial Cátedra, Madrid.

Daly, C., Neilson, R.P. \& Phillips, D.P., 1994. A statisticall-topographic model for mapping climatological precipitation over mountainous terrain. Journal of Applied Meteorology and Climatology, 33: 140-158. Doi: 10.1175/1520-0450(1994)033<0140:ASTMFM>2.0.CO;2.

Dorman, C.E., Beardsley, R.C. \& Limeburner, R., 1995. Winds in the Strait of Gibraltar. Journal of the Royal Meteorological Society, 121: 1903-1921. Doi: 10.1256/smsqj.52806.

Draper Munt, D., Muñoz-Rodríguez, P., Marques, I. \& Moreno Saiz, J.C., 2016. Effects of climate change on threatened Spanish medicinal and aromatic species: predicting future trends and defining conservation guidelines. Israel Journal of Plant Sciences, 63:309-319. Doi: 10.1080/07929978.2016.1258257.

Environmental Systems Research Institute (ESRI), 2016. ArcGIS Desktop: Release 10.5. Redlands, CA: Environmental Systems Research Institute.

Gavira, O.N., 2006. Análisis de un corredor florístico entre los Parques Naturales Sierra de Grazalema y Sierra de las Nieves (Cádiz y Málaga, España). Anales de Biología, 28: 45-72.

Google Earth, 2017. Disponible en: https://earth.google.com/ web/. (Consultado: 10/08/2017).

Goovaerts, P., 2000. Geostatistical approaches for incorporating elevation into the spatial interpolation of rainfall. Journal of Hidrology, 228: 113-129. Doi: 10.1016/ S0022-1694(00)00144-X.

Grosvenor, D.P., King, J.C., Choularton, T.W. \& Lachlan-Cope, T., 2014. Downslope föhn winds over the Antarctic Peninsula and their effect on the Larsen ice shelves. Atmospheric Chemistry and Physics, 14: 9481-9509. Doi: 10.5194/ acp-14-9481-2014.

IBM Corp. Released, 2014. IBM SPSS Statistics for Windows, Version 23.0. Armonk, NY: IBM Corp.

Imeson, A.C. \& Lavee, H., 1998. Soil erosion and climate change: the transect approach and the influence of scale. Geomorphology, 23: 219-227. Doi: 10.1016/S0169-555X(98)00005-1.

Instituto de Estadística y Cartografía de Andalucía (IECA). Datos espaciales de referencia de Andalucía (DERA). Disponible en: http://www.juntadeandalucia.es/institutodeestadisticaycartografia/DERA/index.htm. (Consultado: 21/02/2016). 
Instituto Geográfico Nacional (IGN). Hojas 1036-3, 1049-2 y 4 , 1050-1 a 4, y 1064-1 a 4 del Mapa Topográfico Nacional de España, escala 1:25.000 ráster. Disponible en: http://www. ign.es/csw-inspire/srv/spa/conjuntodatos. (Consultado: 21/02/2016).

Instituto Geográfico Nacional (IGN), 2011. Hoja de Cádiz del Mapa Provincial de España, escala 1:200.000 ráster $\left(10^{\mathrm{a}}\right.$ ed.). Disponible en: http://www.ign.es/csw-inspire/srv/spa/ conjuntodatos. (Consultado: 21/02/2016).

Izquierdo, T. \& Márquez, A., 2007. Estudio comparativo de diferentes métodos de interpolación para la realización de mapas de precipitación en la isla de La Gomera (Islas Canarias): evaluación de la incorporación de la altura. Geogaceta, 42: 131-134.

Junta de Andalucía, 1996. Sistema de información ambiental de Andalucía (SINAMBA) [CD-ROM]. Consejería de Medio Ambiente de la Junta de Andalucía, Sevilla.

Junta de Andalucía, 2006. Plan de Desarrollo Sostenible del Parque Natural Sierra de Grazalema, Decreto 89/2006, de 18 de abril. Disponible en: http://www.juntadeandalucia.es/ medioambiente/site/portalweb/menuitem.7e1cf46ddf59bb2 $27 \mathrm{a} 9 \mathrm{ebe} 205510 \mathrm{e} 1 \mathrm{ca} /$ ? vgnextoid $=3 \mathrm{~b} 41501 \mathrm{f} 8498 \mathrm{~b} 010 \mathrm{VgnV}$ CM1000000624e50aRCRD\&vgnextchannel $=2303$ ee $9 \mathrm{~b} 421$ f4310VgnVCM2000000624e50aRCRD\&lr=lang_es. (Consultado: $21 / 03 / 16$ )

Junta de Andalucía, 2010. Medio ambiente de Andalucía. Informe 2010 [CD-ROM], Consejería de Medio Ambiente de la Junta de Andalucía, Sevilla.

Keblouti, M., Ouerdachi, L. \& Boutaghane, H., 2012. Spatial interpolation of annual precipitation in Annaba-Algeria Comparison and evaluation of methods. Energy Procedia, 18: 468-475. https://doi.org/10.1016/j.egypro.2012.05.058.

Kurtzman, D. \& Kadmon, R., 1999. Mapping of temperature variables in Israel: comparison of different interpolation methods. Climate Research, 13: 33-43.

López-Muñoz, L., Martín-García, E. \& Avila-Rivas, F., 1989. Estudio de una situación atmosférica con intensa circulación zonal en la Sierra de Grazalema (Cádiz). Disponible en: https://repositorio.aemet.es/bitstream/20.500.11765/3546/1/ 1Sim_Pred_01.pdf.

Martín, J., 1991. Rasgos singulares de la pluviometría gaditana. Cuadernos de geografía y ordenación del territorio de la Universidad de Cádiz, 2: 11-20.

Molina-Venegas, R., Aparicio, A., Lavergne, S. \& Arroyo, J., 2017. Climatic and topographical correlates of plant palaeoand neoendemism in a Mediterranean biodiversity hotspot. Annals of Botany, 119: 229-238. Doi: 10.1093/aob/mcw093.

Monkhouse, F.J., 1978: Diccionario de Términos Geográficos. Ed. Oikos-Tau, Barcelona.

Mott, R., 2006. Mecánica de fluidos ( $6^{\mathrm{a}}$ ed.). Pearson Educación, México.

Nastos, P.T., Bleta, A.G. \& Matsangouras, I.T., 2017. Human thermal perception related to Föhn winds due to Saharan dust outbreaks in Crete Island, Greece. Theoretical and Applied Climatology, 128: 635-647. Doi: 10.1007/ s00704-015-1724-3.

Pita, M.F., 2003. El clima de Andalucía, en López Ontiveros, A. (Coord): Geografia de Andalucía., Ariel, 137-174 pp. Barcelona.
Quereda, J., 2005. Curso de climatología general, Publicaciones de la Universitat Jaume I, D.L., Castellón de la Plana.

Ratcliffe, S., Liebergesell, M., Ruiz-Benito, P., Madrigal Gonzalez, J., Castaneda, J.M.M., Kaendler, G., Lehtonen, A., Dahlgren, J., Kattge, J., Penuelas, J., Zavala, M.A. \& Wirth, C., 2016. Modes of functional biodiversity control on tree productivity across the European continent. Global Ecology and Biogeography, 25: 251-262. Doi: 10.1111/geb.12406.

Red de Información Ambiental de Andalucía (REDIAM), 2006. Servicio WMS (Web Map Service) mapa de orientaciones de 100x100 m de Andalucía (para escalas menores a 1:200.000). Disponible en: http://www.juntadeandalucia.es/ medioambiente/site/rediam/menuitem.04dc44281e5d53cf8 ca78ca731525ea $0 /$ ?vgnextoid=0efc 99 afaef $93410 \mathrm{VgnVCM}$ $1000001325 \mathrm{e} 50 \mathrm{aRCRD} \&$ vgnextchannel $=361422 \mathrm{ad} 8470 \mathrm{f} 2$ 10VgnVCM1000001325e50aRCRD\&vgnextfmt=rediam\&1 $\mathrm{r}=$ lang es. (Consultado: 21/02/2016).

Red de Información Ambiental de Andalucía (REDIAM). Subsistema de información de climatología ambiental (CLIMA). Disponible en: http://www.juntadeandalucia.es/ medioambiente/servtc5/WebClima/consulta_avanzada.jsp. (Consultado: 24/09/15 - 09/11/15).

Rivas, S., 1983. Pisos bioclimáticos de España. Lazaroa, 5: 33-43.

Ruiz-Sinoga, J.D., Gabarrón Galeote, M.A., Martínez Murillo, J.F., García Marín, R., 2011. Vegetation strategies for soil water consumption along a pluviometric gradient in southern Spain. Catena, 84: 12-20. Doi: 10.1016/j. catena.2010.08.011.

Senciales, J.M., 1995. La cuenca del Río Vélez. Estudio Hidrográfico-Universidad de Málaga. Tesis Doctoral. Servicio de Publicaciones de la Universidad de Málaga. Ed. Microfichas.

Senciales, J.M. \& Ruiz-Sinoga, J.D., 2013. Análisis espaciotemporal de las lluvias torrenciales en la ciudad de Málaga. Boletín de la Asociación de Geógrafos Españoles, 61: 7-24.

Tabios III, G.Q. \& Salas, J.D., 1985. A comparative analysis of techniques for spatial interpolation of precipitation. Journal of the American Water Resources Association, 21: 365-380. Doi: 10.1111/j.1752-1688.1985.tb00147.x

Van Drooge, B.L. \& Ballesta, P.P., 2010. The influence of the North-Fohn on tracer organic compounds in ambient air PM10 at a pre-alpine site in Northern Italy. Environmental Pollution, 158: 2880-2887. Doi: 10.1016/j.envpol.2010.06.016.

Vázquez, C., 2010. Guía oficial del Parque Natural Sierra de Grazalema. Almuzara - Consejería de Medio Ambiente. Junta de Andalucía, Jaén.

Vicente-Serrano, S.M., Saz-Sánchez, M.A., 2002. Cartografía de precipitaciones y temperaturas en el valle medio del Ebro mediante la utilización de diferentes técnicas estadísticas. Geographicalia, 42: 73-92.

Wetterzentrale, 2017. Top Karten. Disponible en: http://www. wetterzentrale.de/topkarten/fsraeur/html.

Zimmerman, D., Pavlik, C., Ruggles, A. \& Armstrong, P., 1999. An experimental comparison of ordinary and universal kriging and inverse distance weighting. Mathematical Geology, 31: 375-390. Doi: 10.1023/A:1007586507433.

Zúñiga, I., Crespo del Arco, E., 2010. Meteorología y climatologia. Editorial UNED, Madrid. 